\title{
Experimental and theoretical study of model ladder fluoranthenopyracylene with two-dimensional $\pi$-conjugation upon charging: Structure and optical properties
}

Vladimír Lukeš* ${ }^{1}$, Katarína Matuszná ${ }^{1}$, Peter Rapta ${ }^{1,2}$, Roland Šolc ${ }^{1}$, Lothar Dunsch ${ }^{2}$, Adélia Justina Aguiar Aquino ${ }^{3}$ and Hans Lischka ${ }^{3}$

\footnotetext{
${ }^{1}$ Institute of Physical Chemistry and Chemical Physics, Slovak University of Technology, Radlinského 9, SK-81 237 Bratislava, Slovakia

${ }^{2}$ Leibniz Institute for Solid State and Materials Research, Dresden, Helmholtzstrasse 20, D-010 69 Dresden, Germany

${ }^{3}$ Institute for Theoretical Chemistry, University of Vienna, Währingerstrasse 17, A-1090

Wien, Austria
}

*corresponding author: vladimir.lukes@stuba.sk 
Optimized Cartesian geometries in $\AA$ and total energies (E) in Hartree

\section{A conformation, electronic ground state, neutral form, B3LYP/SV(P)}

\section{( $D_{2}$ symmetry)}

\begin{tabular}{|c|c|c|c|}
\hline C & -2.483310 & 2.832287 & 6.563454 \\
\hline $\mathrm{C}$ & -1.086272 & 2.720672 & 6.662912 \\
\hline C & -0.344154 & 3.863950 & 6.99985 \\
\hline C & -0.979730 & 5.089244 & 7.21591 \\
\hline C & -2.372181 & 5.188800 & 7.11492 \\
\hline C & -3.122830 & 4.055450 & 6.7880 \\
\hline C & -0.456385 & 1.362967 & 6.5548 \\
\hline $\mathrm{C}$ & -0.149123 & ๑. 712864 & 5.3007 \\
\hline C & ○. 149123 & -0.712864 & 5.300 \\
\hline C & ๑. 456385 & -1.362967 & 6.5548 \\
\hline C & 0.276371 & -0.666127 & 7.7410 \\
\hline C & -0.276371 & ๑. 666127 & 7.74 \\
\hline $\mathrm{C}$ & -0.133198 & 1.433442 & 4.04367 \\
\hline C & -0.028403 & ๑. 726534 & 2.85 \\
\hline C & ๑. . 028403 & -0.726534 & 2.85811 \\
\hline C & ๑. 133198 & -1.433442 & 4.0436 \\
\hline C & ๑. 508158 & -1.062536 & 9.1513 \\
\hline $\mathrm{C}$ & $\odot .0000 \odot \odot$ & ๑ . ०००००० & 9.94723 \\
\hline C & -0.508158 & 1.062536 & 9.15136 \\
\hline C & 0.006902 & -1.190885 & 1.44525 \\
\hline C & $\odot .0 \odot \odot \odot \odot \odot$ & ๑. . & ๑. 68698 \\
\hline C & -0.006902 & 1.190885 & 1.44525 \\
\hline C & ๑. .000000 & ๑. . 000000 & -0.68698 \\
\hline C & ○. . 006902 & 1.190885 & -1.44525 \\
\hline C & 0.002785 & 2.384753 & -0.71712 \\
\hline C & -0.002785 & 2.384753 & 0.71712 \\
\hline C & -0.006902 & -1.190885 & -1.44525 \\
\hline C & -0.002785 & -2.384753 & -0.71712 \\
\hline C & 0.002785 & -2.384753 & 0.71712 \\
\hline C & $\odot .028403$ & $\odot .726534$ & -2.85811 \\
\hline C & -0.028403 & -0.726534 & -2.85811 \\
\hline C & -0.133198 & -1.433442 & -4.04367 \\
\hline C & -0.149123 & -0.712864 & -5.30079 \\
\hline C & ○. 149123 & ๑. 712864 & $-5.3007 c$ \\
\hline C & ๑. 133198 & 1.433442 & -4.0436 \\
\hline
\end{tabular}




\begin{tabular}{|c|c|c|c|}
\hline$C$ & -0.456385 & -1.362967 & -6.554817 \\
\hline$C$ & -0.276371 & -0.666127 & -7.741033 \\
\hline$C$ & ๑. 276371 & ๑. 666127 & -7.74103 \\
\hline C & ๑. 456385 & 1.362967 & -6.55481 \\
\hline C & -0.508158 & -1.062536 & -9.15130 \\
\hline$C$ & 0.000000 & 0.000000 & -9.94723 \\
\hline$C$ & ๑. 508158 & 1.062536 & -9.15130 \\
\hline$C$ & $\odot .000000$ & $\odot .000000$ & -11.35768 \\
\hline$C$ & 0.580868 & 1.134392 & -11.99381 \\
\hline C & 1.104242 & 2.163733 & -11.22745 \\
\hline$C$ & 1.073985 & 2.149602 & -9.80463 \\
\hline$C$ & -0.580868 & -1.134392 & -11.99381 \\
\hline$C$ & -1.104242 & -2.163733 & -11.22745 \\
\hline$C$ & -1.073985 & -2.149602 & -9.80463 \\
\hline$C$ & $\odot . ๑ \odot \odot \odot \odot \odot$ & $\odot . ๑ \odot \odot \odot \odot \odot$ & 11.35768 \\
\hline$C$ & ๑. 580868 & -1.134392 & 11.99381 \\
\hline$C$ & 1.104242 & -2.163733 & 11.22745 \\
\hline C & 1.073985 & -2.149602 & 9.80463 \\
\hline$C$ & $-\odot .580868$ & 1.134392 & 11.99381 \\
\hline$C$ & -1.104242 & 2.163733 & 11.22745 \\
\hline$C$ & -1.073985 & 2.149602 & 9.80463 \\
\hline C & 1. 086272 & -2.720672 & 6.66291 \\
\hline$C$ & 2.483310 & -2.832287 & 6.56345 \\
\hline$C$ & 3.122830 & -4.055450 & 6.78809 \\
\hline$C$ & 2.372181 & -5.188800 & 7.11492 \\
\hline$C$ & ๑. 979730 & -5.089244 & 7.21591 \\
\hline C & ○. 344154 & -3.863950 & 6.99985 \\
\hline$C$ & $-\odot .071977$ & 2.929165 & 3.92856 \\
\hline$C$ & -1.192492 & 3.708410 & 3.60061 \\
\hline$C$ & -1.067353 & 5.082824 & 3.38216 \\
\hline C & ๑. 185172 & 5.700532 & 3.47089 \\
\hline$C$ & 1.311214 & 4.932838 & 3.78392 \\
\hline C & 1.181506 & 3.559294 & 4.01044 \\
\hline$C$ & ○. . 071977 & -2.929165 & 3.92856 \\
\hline$C$ & -1.181506 & -3.559294 & 4.01044 \\
\hline$C$ & -1.311214 & -4.932838 & 3.78392 \\
\hline$C$ & $-\odot .185172$ & -5.700532 & 3.47089 \\
\hline C & 1.067353 & -5.082824 & 3.38216 \\
\hline$C$ & 1.192492 & -3.708410 & 3.60061 \\
\hline$C$ & ○. . 071977 & 2.929165 & -3.92856 \\
\hline C & -1.181506 & 3.559294 & -4.01044 \\
\hline C & -1.311214 & 4.932838 & -3.78392 \\
\hline C & -0.185172 & 5.700532 & -3.47089 \\
\hline$C$ & 1.067353 & 5.082824 & -3.38216 \\
\hline C & 1.192492 & 3.708410 & -3.60061 \\
\hline C & $-\odot .071977$ & -2.929165 & -3.92856 \\
\hline C & -1.192492 & -3.708410 & -3.60061 \\
\hline
\end{tabular}




\begin{tabular}{|c|c|c|c|}
\hline C & -1.067353 & -5.082824 & -3.382160 \\
\hline C & ๑. 185172 & -5.700532 & -3.470891 \\
\hline C & 1. 311214 & -4.932838 & -3.783923 \\
\hline C & 1.181506 & -3.559294 & -4.010449 \\
\hline C & 1.086272 & 2.720672 & -6.662912 \\
\hline C & ○. 344154 & 3.863950 & -6.999851 \\
\hline C & $\odot .979730$ & 5.089244 & -7.215911 \\
\hline C & 2.372181 & 5.188800 & -7.114927 \\
\hline C & 3.122830 & 4.055450 & -6.788091 \\
\hline C & 2.483310 & 2.832287 & -6.563454 \\
\hline C & -1.086272 & -2.720672 & -6.662912 \\
\hline C & -2.483310 & -2.832287 & -6.563454 \\
\hline C & -3.122830 & -4.055450 & -6.788091 \\
\hline C & -2.372181 & -5.188800 & -7.114927 \\
\hline C & -0.979730 & -5.089244 & -7.215911 \\
\hline C & -0.344154 & -3.863950 & -6.999851 \\
\hline $\mathrm{H}$ & -1.555628 & -3.026530 & -11.727606 \\
\hline $\mathrm{H}$ & -1.499504 & -2.992918 & -9.261776 \\
\hline $\mathrm{H}$ & -0.614835 & -1.187204 & -13.086847 \\
\hline $\mathrm{H}$ & 0.614835 & 1.187204 & -13.086847 \\
\hline $\mathrm{H}$ & 1.499504 & 2.992918 & -9.261776 \\
\hline $\mathrm{H}$ & -0.002735 & -3.353838 & -1.217807 \\
\hline $\mathrm{H}$ & 0.002735 & -3.353838 & 1.217807 \\
\hline $\mathrm{H}$ & 0.002735 & 3.353838 & -1.217807 \\
\hline $\mathrm{H}$ & -0.002735 & 3.353838 & 1.217807 \\
\hline $\mathrm{H}$ & 1.555628 & 3.026530 & -11.727606 \\
\hline $\mathrm{H}$ & 1.499504 & -2.992918 & 9.261776 \\
\hline $\mathrm{H}$ & 1.555628 & -3.026530 & 11.727606 \\
\hline $\mathrm{H}$ & 0.614835 & -1.187204 & 13.086847 \\
\hline $\mathrm{H}$ & -1.499504 & 2.992918 & 9.261776 \\
\hline $\mathrm{H}$ & -1.555628 & 3.026530 & 11.727606 \\
\hline $\mathrm{H}$ & -0.614835 & 1.187204 & 13.086847 \\
\hline $\mathrm{H}$ & -3.075258 & -1.944505 & -6.320231 \\
\hline $\mathrm{H}$ & -4.213072 & -4.121030 & -6.712557 \\
\hline $\mathrm{H}$ & -2.870294 & -6.147235 & -7.292490 \\
\hline $\mathrm{H}$ & -0.383943 & -5.971601 & -7.469681 \\
\hline $\mathrm{H}$ & 0.742435 & -3.789861 & -7.093620 \\
\hline $\mathrm{H}$ & -2.171987 & -3.230784 & -3.515470 \\
\hline $\mathrm{H}$ & 2.066932 & -2.959702 & -4.242202 \\
\hline $\mathrm{H}$ & 2.297921 & -5.403391 & -3.845919 \\
\hline $\mathrm{H}$ & 0.283104 & -6.776114 & -3.292359 \\
\hline $\mathrm{H}$ & -1.954165 & -5.674978 & -3.135019 \\
\hline $\mathrm{H}$ & -0.742435 & 3.789861 & -7.093620 \\
\hline $\mathrm{H}$ & ○. 383943 & 5.971601 & -7.469681 \\
\hline $\mathrm{H}$ & 2.870294 & 6.147235 & -7.292490 \\
\hline $\mathrm{H}$ & 4.213072 & 4.121030 & -6.712557 \\
\hline $\mathrm{H}$ & 3.075258 & 1.944505 & -6.320231 \\
\hline
\end{tabular}




$\begin{array}{rrrr}\mathrm{H} & -2.066932 & 2.959702 & -4.242202 \\ \mathrm{H} & -2.297921 & 5.403391 & -3.845919 \\ \mathrm{H} & -0.283104 & 6.776114 & -3.292359 \\ \mathrm{H} & 1.954165 & 5.674978 & -3.135019 \\ \mathrm{H} & 2.171987 & 3.230784 & -3.515470 \\ \mathrm{H} & -2.171987 & 3.230784 & 3.515470 \\ \mathrm{H} & -1.954165 & 5.674978 & 3.135019 \\ \mathrm{H} & 0.283104 & 6.776114 & 3.292359 \\ \mathrm{H} & 2.297921 & 5.403391 & 3.845919 \\ \mathrm{H} & 2.066932 & 2.959702 & 4.242202 \\ \mathrm{H} & 0.742435 & 3.789861 & 7.093620 \\ \mathrm{H} & -0.383943 & 5.971601 & 7.469681 \\ \mathrm{H} & -2.870294 & 6.147235 & 7.292490 \\ \mathrm{H} & -4.213072 & 4.121030 & 6.712557 \\ \mathrm{H} & -3.075258 & 1.944505 & 6.320231 \\ \mathrm{H} & -2.066932 & -2.959702 & 4.242202 \\ \mathrm{H} & -2.297921 & -5.403391 & 3.845919 \\ \mathrm{H} & -0.283104 & -6.776114 & 3.292359 \\ \mathrm{H} & 1.954165 & -5.674978 & 3.135019 \\ \mathrm{H} & 2.171987 & -3.230784 & 3.515470 \\ \mathrm{H} & -0.742435 & -3.789861 & 7.093620 \\ \mathrm{H} & 0.383943 & -5.971601 & 7.469681 \\ \mathrm{H} & 2.870294 & -6.147235 & 7.292490 \\ \mathrm{H} & 4.213072 & -4.121030 & 6.712557 \\ \mathrm{H} & 3.075258 & -1.944505 & 6.320231\end{array}$

A conformation, electronic ground state, neutral form, ONIOMB3LYP/SV (P)

\section{( $D_{2}$ symmetry)}

\begin{tabular}{|c|c|c|c|}
\hline$C$ & -2.483310 & 2.832287 & 6.563454 \\
\hline$C$ & -1.086272 & 2.720672 & 6.662912 \\
\hline$C$ & -0.344154 & 3.863950 & 6.999851 \\
\hline$C$ & $-\odot .979730$ & 5.089244 & 7.215911 \\
\hline$C$ & -2.372181 & 5.188800 & 7.114927 \\
\hline$C$ & -3.122830 & 4.055450 & 6.788091 \\
\hline$C$ & $-\odot .456385$ & 1.362967 & 6.554817 \\
\hline$C$ & -0.149123 & ๑. 712864 & 5.300793 \\
\hline$C$ & ๑. 149123 & -0.712864 & 5.300793 \\
\hline C & ๑. 456385 & -1.362967 & 6.554817 \\
\hline C & ๑. 276371 & -0.666127 & 7.741033 \\
\hline C & -0.276371 & ๑. 666127 & 7.741033 \\
\hline
\end{tabular}




\begin{tabular}{|c|c|c|c|}
\hline C & -0.133198 & 1.433442 & 4.043673 \\
\hline C & -0.028403 & 0.726534 & 2.858118 \\
\hline C & ๑. . 028403 & -0.726534 & 2.858118 \\
\hline C & ๑. 133198 & -1.433442 & 4.043673 \\
\hline C & ๑. 508158 & -1.062536 & 9.151303 \\
\hline C & 0.000000 & 0.000000 & 9.947233 \\
\hline C & -0.508158 & 1.062536 & 9.151303 \\
\hline C & ๑. . 006902 & -1.190885 & 1. 445251 \\
\hline C & 0.000000 & 0.000000 & ๑. 686986 \\
\hline C & -0.006902 & 1.190885 & 1. 445251 \\
\hline C & ๑. . 000000 & ๑ . $00000 \odot$ & -0.686986 \\
\hline C & ๑. . 006902 & 1.190885 & -1.445251 \\
\hline C & 0.002785 & 2.384753 & -0.717125 \\
\hline C & -0.002785 & 2.384753 & 0.717125 \\
\hline C & -0.006902 & -1.190885 & -1.445251 \\
\hline C & -0.002785 & -2.384753 & -0.717125 \\
\hline C & 0.002785 & -2.384753 & 0.717125 \\
\hline C & ๑. . 028403 & 0.726534 & -2.858118 \\
\hline C & -0.028403 & -0.726534 & -2.858118 \\
\hline C & -0.133198 & -1.433442 & -4.043673 \\
\hline C & -0.149123 & -0.712864 & -5.300793 \\
\hline C & 0.149123 & 0.712864 & -5.300793 \\
\hline C & 0.133198 & 1.433442 & -4.043673 \\
\hline C & -0.456385 & -1.362967 & -6.554817 \\
\hline C & -0.276371 & -0.666127 & -7.741033 \\
\hline C & ๑. 276371 & ๑. 666127 & -7.741033 \\
\hline C & ๑. 456385 & 1.362967 & -6.554817 \\
\hline C & -0.508158 & -1.062536 & -9.151303 \\
\hline C & ०. . 000000 & ๑ . . 000000 & -9.947233 \\
\hline C & ๑. 508158 & 1.062536 & -9.151303 \\
\hline C & $\odot .000000$ & $\odot . \odot \odot \odot \odot \odot \odot$ & -11.357680 \\
\hline C & ๑. 580868 & 1.134392 & -11.993814 \\
\hline C & 1.104242 & 2.163733 & -11.227450 \\
\hline C & 1.073985 & 2.149602 & -9.804637 \\
\hline C & -0.580868 & -1.134392 & -11.993814 \\
\hline C & -1.104242 & -2.163733 & -11.227450 \\
\hline C & -1.073985 & -2.149602 & -9.804637 \\
\hline C & ๑. . 000000 & ๑ . 000000 & 11.357680 \\
\hline C & 0.580868 & -1.134392 & 11.993814 \\
\hline C & 1.104242 & -2.163733 & 11.227450 \\
\hline C & 1.073985 & -2.149602 & 9.804637 \\
\hline C & -0.580868 & 1.134392 & 11.993814 \\
\hline C & -1.104242 & 2.163733 & 11.227450 \\
\hline C & -1.073985 & 2.149602 & 9.804637 \\
\hline C & 1.086272 & -2.720672 & 6.662912 \\
\hline
\end{tabular}




\begin{tabular}{|c|c|c|c|}
\hline$C$ & 2.483310 & -2.832287 & 6.563454 \\
\hline$C$ & 3.122830 & -4.055450 & 6.788091 \\
\hline$C$ & 2.372181 & -5.188800 & 7.114927 \\
\hline$C$ & ๑. 979730 & -5.089244 & 7.215911 \\
\hline$C$ & ๑. 344154 & -3.863950 & 6.999851 \\
\hline$C$ & -0.071977 & 2.929165 & 3.928563 \\
\hline$C$ & -1.192492 & 3.708410 & 3.600614 \\
\hline$C$ & -1.067353 & 5.082824 & 3.382160 \\
\hline$C$ & ๑. 185172 & 5.700532 & 3.470891 \\
\hline$C$ & 1.311214 & 4.932838 & 3.783923 \\
\hline$C$ & 1.181506 & 3.559294 & 4.010449 \\
\hline$C$ & ๑ . 071977 & -2.929165 & 3.928563 \\
\hline$C$ & -1.181506 & -3.559294 & 4.010449 \\
\hline$C$ & -1.311214 & -4.932838 & 3.783923 \\
\hline$C$ & $-\odot .185172$ & -5.700532 & 3.470891 \\
\hline$C$ & 1.067353 & -5.082824 & 3.382160 \\
\hline$C$ & 1.192492 & -3.708410 & 3.600614 \\
\hline$C$ & ๑. 071977 & 2.929165 & -3.928563 \\
\hline$C$ & -1.181506 & 3.559294 & -4.010449 \\
\hline$C$ & -1.311214 & 4.932838 & -3.783923 \\
\hline$C$ & $-\odot .185172$ & 5.700532 & -3.470891 \\
\hline$C$ & 1.067353 & 5.082824 & -3.382160 \\
\hline$C$ & 1.192492 & 3.708410 & -3.600614 \\
\hline$C$ & $-\odot .071977$ & -2.929165 & -3.928563 \\
\hline$C$ & -1.192492 & -3.708410 & -3.600614 \\
\hline$C$ & -1.067353 & -5.082824 & -3.382160 \\
\hline$C$ & ๑. 185172 & -5.700532 & -3.470891 \\
\hline$C$ & 1.311214 & -4.932838 & -3.783923 \\
\hline C & 1.181506 & -3.559294 & -4.010449 \\
\hline$C$ & 1.086272 & 2.720672 & -6.662912 \\
\hline$C$ & ๑. 344154 & 3.863950 & -6.999851 \\
\hline$C$ & ๑. 979730 & 5.089244 & -7.215911 \\
\hline$C$ & 2.372181 & 5.188800 & -7.114927 \\
\hline$C$ & 3.122830 & 4.055450 & -6.788091 \\
\hline$C$ & 2.483310 & 2.832287 & -6.563454 \\
\hline$C$ & -1.086272 & -2.720672 & -6.662912 \\
\hline$C$ & -2.483310 & -2.832287 & -6.563454 \\
\hline$C$ & -3.122830 & -4.055450 & -6.788091 \\
\hline$C$ & -2.372181 & -5.188800 & -7.114927 \\
\hline$C$ & $-\odot .979730$ & -5.089244 & -7.215911 \\
\hline C & -0.344154 & -3.863950 & -6.999851 \\
\hline $\mathrm{H}$ & -1.555628 & -3.026530 & -11.727606 \\
\hline $\mathrm{H}$ & -1.499504 & -2.992918 & -9.261776 \\
\hline $\mathrm{H}$ & -0.614835 & -1.187204 & -13.086847 \\
\hline $\mathrm{H}$ & ๑. 614835 & 1.187204 & -13.086847 \\
\hline
\end{tabular}




\begin{tabular}{|c|c|c|c|}
\hline $\mathrm{H}$ & 1.499504 & 2.992918 & -9.261776 \\
\hline $\mathrm{H}$ & -0.002735 & -3.353838 & -1.217807 \\
\hline $\mathrm{H}$ & 0.002735 & -3.353838 & 1.217807 \\
\hline $\mathrm{H}$ & ๑ . 002735 & 3.353838 & -1.217807 \\
\hline $\mathrm{H}$ & $-\odot .002735$ & 3.353838 & 1.217807 \\
\hline $\mathrm{H}$ & 1.555628 & 3.026530 & -11.727606 \\
\hline $\mathrm{H}$ & 1.499504 & -2.992918 & 9.261776 \\
\hline $\mathrm{H}$ & 1.555628 & -3.026530 & 11.727606 \\
\hline $\mathrm{H}$ & $\odot .614835$ & -1.187204 & 13.086847 \\
\hline $\mathrm{H}$ & -1.499504 & 2.992918 & 9.261776 \\
\hline $\mathrm{H}$ & -1.555628 & 3.026530 & 11.727606 \\
\hline $\mathrm{H}$ & -0.614835 & 1.187204 & 13.086847 \\
\hline $\mathrm{H}$ & -3.075258 & -1.944505 & -6.320231 \\
\hline $\mathrm{H}$ & -4.213072 & -4.121030 & -6.712557 \\
\hline $\mathrm{H}$ & -2.870294 & -6.147235 & -7.292490 \\
\hline $\mathrm{H}$ & -0.383943 & -5.971601 & -7.469681 \\
\hline $\mathrm{H}$ & 0.742435 & -3.789861 & -7.093620 \\
\hline $\mathrm{H}$ & -2.171987 & -3.230784 & -3.515470 \\
\hline $\mathrm{H}$ & 2.066932 & -2.959702 & -4.242202 \\
\hline $\mathrm{H}$ & 2.297921 & -5.403391 & -3.845919 \\
\hline $\mathrm{H}$ & ๑. 283104 & -6.776114 & -3.292359 \\
\hline $\mathrm{H}$ & -1.954165 & -5.674978 & -3.135019 \\
\hline $\mathrm{H}$ & -0.742435 & 3.789861 & -7.093620 \\
\hline $\mathrm{H}$ & ๑. 383943 & 5.971601 & -7.469681 \\
\hline $\mathrm{H}$ & 2.870294 & 6.147235 & -7.292490 \\
\hline $\mathrm{H}$ & 4.213072 & 4.121030 & -6.712557 \\
\hline $\mathrm{H}$ & 3.075258 & 1.944505 & -6.320231 \\
\hline $\mathrm{H}$ & -2.066932 & 2.959702 & -4.242202 \\
\hline $\mathrm{H}$ & -2.297921 & 5.403391 & -3.845919 \\
\hline $\mathrm{H}$ & -0.283104 & 6.776114 & -3.292359 \\
\hline $\mathrm{H}$ & 1.954165 & 5.674978 & -3.135019 \\
\hline $\mathrm{H}$ & 2.171987 & 3.230784 & -3.515470 \\
\hline $\mathrm{H}$ & -2.171987 & 3.230784 & 3.515470 \\
\hline $\mathrm{H}$ & -1.954165 & 5.674978 & 3.135019 \\
\hline $\mathrm{H}$ & ๑. 283104 & 6.776114 & 3.292359 \\
\hline $\mathrm{H}$ & 2.297921 & 5.403391 & 3.845919 \\
\hline $\mathrm{H}$ & 2.066932 & 2.959702 & 4.242202 \\
\hline $\mathrm{H}$ & 0.742435 & 3.789861 & 7.093620 \\
\hline $\mathrm{H}$ & -0.383943 & 5.971601 & 7.469681 \\
\hline $\mathrm{H}$ & -2.870294 & 6.147235 & 7.292490 \\
\hline $\mathrm{H}$ & -4.213072 & 4.121030 & 6.712557 \\
\hline $\mathrm{H}$ & -3.075258 & 1.944505 & 6.320231 \\
\hline $\mathrm{H}$ & -2.066932 & -2.959702 & 4.242202 \\
\hline $\mathrm{H}$ & -2.297921 & -5.403391 & 3.845919 \\
\hline $\mathrm{H}$ & -0.283104 & -6.776114 & 3.292359 \\
\hline
\end{tabular}




$\begin{array}{lrrr}\mathrm{H} & 1.954165 & -5.674978 & 3.135019 \\ \mathrm{H} & 2.171987 & -3.230784 & 3.515470 \\ \mathrm{H} & -0.742435 & -3.789861 & 7.093620 \\ \mathrm{H} & 0.383943 & -5.971601 & 7.469681 \\ \mathrm{H} & 2.870294 & -6.147235 & 7.292490 \\ \mathrm{H} & 4.213072 & -4.121030 & 6.712557 \\ \mathrm{H} & 3.075258 & -1.944505 & 6.320231\end{array}$

\section{A conformation, electronic ground state, neutral form, $\mathrm{HF} / \mathrm{SV}(\mathrm{P})$}

\section{( $D_{2}$ symmetry)}

\begin{tabular}{|c|c|c|c|}
\hline C & 6.911710 & 3.611135 & 1.196453 \\
\hline C & 6.738148 & 2.921714 & ๑ . 000000 \\
\hline C & 6.911710 & 3.611135 & -1.196453 \\
\hline C & 7.242342 & 4.959010 & -1.199561 \\
\hline C & 7.411169 & 5.637615 & 0.000000 \\
\hline C & 7.242342 & 4.959010 & 1.199561 \\
\hline C & 6.563046 & 1.426422 & 0.000000 \\
\hline C & 5.296966 & ๑. 722014 & $\odot .000000$ \\
\hline C & 5.296966 & -0.722014 & $\odot .00000$ \\
\hline C & 6.563046 & -1.426422 & ๑ . $00000 \odot$ \\
\hline C & 7.733337 & -0.715844 & ०. . $00 \odot ० ० \odot$ \\
\hline C & 7.733337 & 0.715844 & 0.00000 \\
\hline C & 4.025908 & -1.429320 & $\odot ., 000 \odot ० ०$ \\
\hline C & 2.859319 & -0.721384 & ๑. . 00000 \\
\hline C & 2.859319 & $\odot .721384$ & $\odot .0 \odot \odot \odot \odot \odot$ \\
\hline C & 4.025908 & 1.429320 & 0.000000 \\
\hline C & 1.434397 & -1.185233 & 0.00000 \\
\hline C & ๑. 681412 & ๑ . ००००००० & ๑ . . 000000 \\
\hline C & 1.434397 & 1.185233 & ०. . 00000 \\
\hline C & -0.681412 & ๑ . $00 \odot ० ० \odot$ & ๑ . $00 \odot ० ० \odot$ \\
\hline C & -1.434397 & 1.185233 & 0.000000 \\
\hline C & -0.717093 & 2.361372 & 0.000000 \\
\hline C & 0.717093 & 2.361372 & 0.000000 \\
\hline C & -1.434397 & -1.185233 & $\odot .0 \odot \odot \odot \odot \odot$ \\
\hline C & -2.859319 & -0.721384 & ๑ . $00000 \odot$ \\
\hline C & -2.859319 & 0.721384 & 0.000000 \\
\hline C & -4.025908 & -1.429320 & 0.00000 \\
\hline C & -5.296966 & -0.722014 & ๑. . 000000 \\
\hline C & -5.296966 & $\odot .722014$ & ๑. $\odot \odot \odot \odot \odot ~$ \\
\hline C & -4.025908 & 1.429320 & 0.000000 \\
\hline C & -6.563046 & -1.426422 & 0.00000 \\
\hline C & -7.733337 & -0.715844 & 0.00000 \\
\hline C & -7.733337 & 0.715844 & 0.00000 \\
\hline
\end{tabular}




\begin{tabular}{|c|c|c|c|}
\hline$C$ & -6.563046 & 1.426422 & ๑ . ๑๑९७०७ \\
\hline$C$ & -9.155894 & -1.175153 & 0.000000 \\
\hline C & -9.945573 & $\odot .000000$ & ๑ . 000000 \\
\hline C & -9.155894 & 1.175153 & ๑. . 000000 \\
\hline$C$ & -9.805987 & 2.381720 & $\odot .000000$ \\
\hline C & -11.228501 & 2.408120 & $\odot .000 \odot \odot \odot$ \\
\hline C & -11.981356 & 1.267982 & 0.000000 \\
\hline C & -11.338786 & ๑ . 000000 & ๑. . $00000 \odot$ \\
\hline C & -9.805987 & -2.381720 & ๑. . 000000 \\
\hline C & -11.228501 & -2.408120 & ๑. .००००८ \\
\hline C & -11.981356 & -1.267982 & $\odot .0 \odot \odot \odot ० \odot$ \\
\hline $\mathrm{C}$ & ๑. 717093 & -2.361372 & $\odot .000000$ \\
\hline C & -0.717093 & -2.361372 & ๑. . 000000 \\
\hline$C$ & 9.155894 & 1.175153 & ๑ . 000000 \\
\hline C & 9.945573 & $\odot .00 \odot \odot \odot \odot$ & ๑ . ๑๑૦००० \\
\hline C & 9.155894 & -1.175153 & ๑ . 000000 \\
\hline C & 11.338786 & ๑ . .00000० & ๑. . 000000 \\
\hline C & 11.981356 & 1. 267982 & $\odot .00000 \odot$ \\
\hline C & 11.228501 & 2.408120 & ๑. .००००८ \\
\hline C & 9.805987 & 2.381720 & ๑ . 000000 \\
\hline C & 11.981356 & -1.267982 & ๑. . $00000 \odot$ \\
\hline C & 11.228501 & -2.408120 & $\odot .00000 \odot$ \\
\hline $\mathrm{C}$ & 9.805987 & -2.381720 & $\odot .000000$ \\
\hline$C$ & -6.738148 & 2.921714 & ๑ . ๑૦૦००७ \\
\hline$C$ & -6.911710 & 3.611135 & -1.196453 \\
\hline C & -7.242342 & 4.959010 & -1.199561 \\
\hline C & -7.411169 & 5.637615 & 0.000000 \\
\hline C & -7.242342 & 4.959010 & 1.199561 \\
\hline C & -6.911710 & 3.611135 & 1.196453 \\
\hline$C$ & -6.738148 & -2.921714 & 0.000000 \\
\hline C & -6.911710 & -3.611135 & -1.196453 \\
\hline C & -7.242342 & -4.959010 & -1.199561 \\
\hline C & -7.411169 & -5.637615 & ๑ . 000000 \\
\hline$C$ & -7.242342 & -4.959010 & 1.199561 \\
\hline$C$ & -6.911710 & -3.611135 & 1.196453 \\
\hline C & -3.834057 & 2.924119 & ๑. . 000000 \\
\hline C & -3.648188 & 3.611066 & 1.196191 \\
\hline C & -3.298377 & 4.954011 & 1.199251 \\
\hline$C$ & -3.120785 & 5.630974 & ๑ . 000000 \\
\hline$C$ & -3.298377 & 4.954011 & -1.199251 \\
\hline C & -3.648188 & 3.611066 & -1.196191 \\
\hline C & -3.834057 & -2.924119 & ๑. . 000000 \\
\hline C & -3.648188 & -3.611066 & 1.196191 \\
\hline$C$ & -3.298377 & -4.954011 & 1.199251 \\
\hline$C$ & -3.120785 & -5.630974 & ๑ . 000000 \\
\hline$C$ & -3.298377 & -4.954011 & -1.199251 \\
\hline$C$ & -3.648188 & -3.611066 & -1.196191 \\
\hline
\end{tabular}




\begin{tabular}{|c|c|c|c|}
\hline C & 3.834057 & 2.924119 & ๑ . . 000000 \\
\hline C & 3.648188 & 3.611066 & -1.196191 \\
\hline C & 3.298377 & 4.954011 & -1.199251 \\
\hline C & 3.120785 & 5.630974 & $\odot . ๑ \odot \odot \odot \odot \odot$ \\
\hline C & 3.298377 & 4.954011 & 1. 199251 \\
\hline C & 3.648188 & 3.611066 & 1.196191 \\
\hline C & 3.834057 & -2.924119 & ๑. . $\odot ० \odot ० \odot$ \\
\hline C & 3.648188 & -3.611066 & 1.196191 \\
\hline C & 3.298377 & -4.954011 & 1.199251 \\
\hline C & 3.120785 & -5.630974 & ๑ . $0000 \odot \odot$ \\
\hline C & 3.298377 & -4.954011 & -1.199251 \\
\hline C & 3.648188 & -3.611066 & -1.196191 \\
\hline C & 6.738148 & -2.921714 & $\odot . ๑ \odot \odot \odot \odot \odot$ \\
\hline C & 6.911710 & -3.611135 & 1.196453 \\
\hline C & 7.242342 & -4.959010 & 1.199561 \\
\hline C & 7.411169 & -5.637615 & ๑. . 000000 \\
\hline C & 7.242342 & -4.959010 & -1.199561 \\
\hline C & 6.911710 & -3.611135 & -1.196453 \\
\hline $\mathrm{H}$ & -11.720757 & 3.372578 & 0.000000 \\
\hline $\mathrm{H}$ & -9.284969 & 3.322393 & ๑ . .०००००० \\
\hline $\mathrm{H}$ & -13.062629 & 1.324527 & $\odot .000000$ \\
\hline $\mathrm{H}$ & -13.062629 & -1.324527 & $\odot . ๑ \odot \odot \odot \odot \odot$ \\
\hline $\mathrm{H}$ & -9.284969 & -3.322393 & 0.000000 \\
\hline $\mathrm{H}$ & -1.198879 & 3.324363 & ๑ . 000000 \\
\hline $\mathrm{H}$ & 1.198879 & 3.324363 & ๑ . . 000000 \\
\hline $\mathrm{H}$ & -1.198879 & -3.324363 & $\odot . ๑ \odot \odot \odot ० \odot$ \\
\hline H & 1.198879 & -3.324363 & $\odot .000000$ \\
\hline $\mathrm{H}$ & -11.720757 & -3.372578 & 0.000000 \\
\hline $\mathrm{H}$ & 9.284969 & 3.322393 & ๑ . . 000000 \\
\hline $\mathrm{H}$ & 11.720757 & 3.372578 & ๑ . . 000000 \\
\hline $\mathrm{H}$ & 13.062629 & 1.324527 & $\odot . ๑ \odot \odot \odot \odot \odot$ \\
\hline $\mathrm{H}$ & 9.284969 & -3.322393 & 0.000000 \\
\hline $\mathrm{H}$ & 11.720757 & -3.372578 & 0.000000 \\
\hline $\mathrm{H}$ & 13.062629 & -1.324527 & ๑ . . 000000 \\
\hline $\mathrm{H}$ & -6.801198 & 3.084149 & -2.135478 \\
\hline $\mathrm{H}$ & -7.374645 & 5.478078 & -2.140361 \\
\hline $\mathrm{H}$ & -7.673483 & 6.688128 & 0.000000 \\
\hline $\mathrm{H}$ & -7.374645 & 5.478078 & 2.140361 \\
\hline $\mathrm{H}$ & -6.801198 & 3.084149 & 2.135478 \\
\hline H & -3.764447 & 3.086197 & 2.135487 \\
\hline H & -3.764447 & 3.086197 & -2.135487 \\
\hline $\mathrm{H}$ & -3.156412 & 5.469569 & -2.140990 \\
\hline $\mathrm{H}$ & -2.843100 & 6.677680 & ๑ . . 000000 \\
\hline $\mathrm{H}$ & -3.156412 & 5.469569 & 2.140990 \\
\hline $\mathrm{H}$ & -6.801198 & -3.084149 & -2.135478 \\
\hline $\mathrm{H}$ & -7.374645 & -5.478078 & -2.140361 \\
\hline $\mathrm{H}$ & -7.673483 & -6.688128 & 0.000000 \\
\hline
\end{tabular}




$\begin{array}{lrrr}\mathrm{H} & -7.374645 & -5.478078 & 2.140361 \\ \mathrm{H} & -6.801198 & -3.084149 & 2.135478 \\ \mathrm{H} & -3.764447 & -3.086197 & 2.135487 \\ \mathrm{H} & -3.156412 & -5.469569 & 2.140990 \\ \mathrm{H} & -2.843100 & -6.677680 & 0.000000 \\ \mathrm{H} & -3.156412 & -5.469569 & -2.140990 \\ \mathrm{H} & -3.764447 & -3.086197 & -2.135487 \\ \mathrm{H} & 3.764447 & -3.086197 & 2.135487 \\ \mathrm{H} & 3.156412 & -5.469569 & 2.140990 \\ \mathrm{H} & 2.843100 & -6.677680 & 0.000000 \\ \mathrm{H} & 3.156412 & -5.469569 & -2.140990 \\ \mathrm{H} & 3.764447 & -3.086197 & -2.135487 \\ \mathrm{H} & 6.801198 & -3.084149 & 2.135478 \\ \mathrm{H} & 7.374645 & -5.478078 & 2.140361 \\ \mathrm{H} & 7.673483 & -6.688128 & 0.000000 \\ \mathrm{H} & 7.374645 & -5.478078 & -2.140361 \\ \mathrm{H} & 6.801198 & -3.084149 & -2.135478 \\ \mathrm{H} & 3.764447 & 3.086197 & -2.135487 \\ \mathrm{H} & 3.156412 & 5.469569 & -2.140990 \\ \mathrm{H} & 2.843100 & 6.677680 & 0.000000 \\ \mathrm{H} & 3.156412 & 5.469569 & 2.140990 \\ \mathrm{H} & 3.764447 & 3.086197 & 2.135487 \\ \mathrm{H} & 6.801198 & 3.084149 & -2.135478 \\ \mathrm{H} & 7.374645 & 5.478078 & -2.140361 \\ \mathrm{H} & 7.673483 & 6.688128 & 0.000000 \\ \mathrm{H} & 7.374645 & 5.478078 & 2.140361 \\ \mathrm{H} & 6.801198 & 3.084149 & 2.135478 \\ & \mathrm{E}=-3740.838217 & \\ & & & \end{array}$

\section{A conformation, lowest electronic ground state, neutral form, TDHF/SV(P)}

\section{( $D_{2}$ symmetry)}

$\begin{array}{lrrr}\text { C } & 6.903226 & 3.610514 & 1.196634 \\ \text { C } & 6.727913 & 2.922103 & 0.000000 \\ \text { C } & 6.903226 & 3.610514 & -1.196634 \\ \text { C } & 7.238106 & 4.957189 & -1.199567 \\ \text { C } & 7.408675 & 5.635554 & 0.00000 \odot \\ \text { C } & 7.238106 & 4.957189 & 1.199567 \\ \text { C } & 6.549193 & 1.427278 & 0.000000 \\ \text { C } & 5.286814 & 0.727181 & 0.00000 \odot \\ \text { C } & 5.286814 & -0.727181 & 0.00000 \odot \\ \text { C } & 6.549193 & -1.427278 & 0.00000 \odot \\ \text { C } & 7.724557 & -0.715172 & 0.00000 \odot\end{array}$




\begin{tabular}{|c|c|c|c|}
\hline C & 7.724557 & 0.715172 & ๑ . ०००००० \\
\hline $\mathrm{C}$ & 4.018819 & -1.432342 & $\odot .0 \odot \odot ० \odot \odot$ \\
\hline C & 2.840801 & -0.726387 & 0.000000 \\
\hline C & 2.840801 & 0.726387 & 0.000000 \\
\hline $\mathrm{C}$ & 4.018819 & 1.432342 & 0.000000 \\
\hline C & 1.441800 & -1.175794 & $\odot .0 \odot \odot \odot \odot \odot$ \\
\hline C & $\odot .692131$ & $\odot .0 \odot \odot \odot \odot \odot$ & ๑. . ०००००० \\
\hline C & 1. 441800 & 1.175794 & 0.000000 \\
\hline C & -0.692131 & 0.000000 & 0.000000 \\
\hline $\mathrm{C}$ & -1.441800 & 1.175794 & 0.000000 \\
\hline C & -0.693230 & 2.394494 & $\odot .0 \odot \odot \odot \odot \odot$ \\
\hline $\mathrm{C}$ & 0.693230 & 2.394494 & 0.000000 \\
\hline $\mathrm{C}$ & -1.441800 & -1.175794 & 0.000000 \\
\hline C & - 2.840801 & -0.726387 & ๑. . 000000 \\
\hline C & -2.840801 & 0.726387 & 0.000000 \\
\hline C & -4.018819 & -1.432342 & ๑. . $૦ \odot ० \odot \odot$ \\
\hline C & -5.286814 & -0.727181 & 0.000000 \\
\hline C & -5.286814 & 0.727181 & 0.000000 \\
\hline C & -4.018819 & 1.432342 & $\odot .0 \odot ० \odot ० \odot$ \\
\hline C & -6.549193 & -1.427278 & $\odot . ๑ \odot \odot \odot \odot \odot$ \\
\hline C & -7.724557 & -0.715172 & 0.000000 \\
\hline C & -7.724557 & 0.715172 & 0.000000 \\
\hline C & -6.549193 & 1.427278 & 0.000000 \\
\hline C & -9.143400 & -1.174620 & ๑. . 000000 \\
\hline C & -9.933867 & ๑ . $000 \odot \odot \odot$ & ๑ . ०૦०००० \\
\hline C & -9.143400 & 1.174620 & 0.000000 \\
\hline C & -9.795701 & 2.382707 & ๑. .0०००००० \\
\hline C & -11.216586 & 2.408328 & 0.000000 \\
\hline C & -11.970308 & 1.267430 & ๑ . $00000 \odot$ \\
\hline C & -11.327834 & $\odot .0 \odot \odot \odot \odot \odot$ & ๑. . $0 \odot \odot ० ० \odot$ \\
\hline C & -9.795701 & -2.382707 & 0.000000 \\
\hline C & -11.216586 & -2.408328 & ๑. . $00 \odot ० ० \odot$ \\
\hline C & -11.970308 & -1.267430 & 0.000000 \\
\hline C & $\odot .693230$ & -2.394494 & $\odot .0 \odot \odot \odot \odot \odot$ \\
\hline C & -0.693230 & -2.394494 & 0.000000 \\
\hline C & 9.143400 & 1.174620 & ๑. . 000000 \\
\hline C & 9.933867 & 0.000000 & 0.000000 \\
\hline C & 9.143400 & -1.174620 & ๑. . 000000 \\
\hline C & 11.327834 & ๑. . 000000 & 0.000000 \\
\hline C & 11.970308 & 1.267430 & 0.000000 \\
\hline C & 11.216586 & 2.408328 & ๑. . $0 \odot \odot ० ० \odot$ \\
\hline $\mathrm{C}$ & 9.795701 & 2.382707 & 0.000000 \\
\hline C & 11.970308 & -1.267430 & $\odot .0 \odot \odot \odot \odot \odot$ \\
\hline C & 11.216586 & -2.408328 & ๑ . . 000000 \\
\hline C & 9.795701 & -2.382707 & 0.000000 \\
\hline C & -6.727913 & 2.922103 & 0.000000 \\
\hline C & -6.903226 & 3.610514 & -1.196634 \\
\hline
\end{tabular}




\begin{tabular}{|c|c|c|c|}
\hline C & -7.238106 & 4.957189 & -1.199567 \\
\hline C & -7.408675 & 5.635554 & ๑. . 000000 \\
\hline C & -7.238106 & 4.957189 & 1.199567 \\
\hline C & -6.903226 & 3.610514 & 1.196634 \\
\hline C & -6.727913 & -2.922103 & ๑. . \\
\hline C & -6.903226 & -3.610514 & -1.196634 \\
\hline C & -7.238106 & -4.957189 & -1.199567 \\
\hline C & -7.408675 & -5.635554 & $\odot . ๑ \odot \odot \odot \odot \odot$ \\
\hline C & -7.238106 & -4.957189 & 1.199567 \\
\hline C & -6.903226 & -3.610514 & 1.196634 \\
\hline C & -3.827482 & 2.925901 & ๑. . $00000 \odot$ \\
\hline C & -3.641838 & 3.611324 & 1.196829 \\
\hline C & -3.292062 & 4.954232 & 1.199420 \\
\hline C & -3.114959 & 5.630786 & 0.000000 \\
\hline C & -3.292062 & 4.954232 & -1.199420 \\
\hline C & -3.641838 & 3.611324 & -1.196829 \\
\hline C & -3.827482 & -2.925901 & ๑. . \\
\hline C & -3.641838 & -3.611324 & 1.196829 \\
\hline C & -3.292062 & -4.954232 & 1.199420 \\
\hline C & -3.114959 & -5.630786 & ๑. . 000000 \\
\hline C & -3.292062 & -4.954232 & -1.199420 \\
\hline C & -3.641838 & -3.611324 & -1.196829 \\
\hline C & 3.827482 & 2.925901 & ๑ . 000000 \\
\hline C & 3.641838 & 3.611324 & -1.196829 \\
\hline C & 3.292062 & 4.954232 & -1.199420 \\
\hline C & 3.114959 & 5.630786 & $\odot .0 \odot \odot ० \odot \odot$ \\
\hline C & 3.292062 & 4.954232 & 1.199420 \\
\hline C & 3.641838 & 3.611324 & 1.196829 \\
\hline C & 3.827482 & -2.925901 & ๑ . 000000 \\
\hline C & 3.641838 & -3.611324 & 1.196829 \\
\hline C & 3.292062 & -4.954232 & 1.199420 \\
\hline $\mathrm{C}$ & 3.114959 & -5.630786 & 0.000000 \\
\hline C & 3.292062 & -4.954232 & -1.199420 \\
\hline C & 3.641838 & -3.611324 & -1.196829 \\
\hline $\mathrm{C}$ & 6.727913 & -2.922103 & ๑. . 000000 \\
\hline C & 6.903226 & -3.610514 & 1.196634 \\
\hline C & 7.238106 & -4.957189 & 1.199567 \\
\hline C & 7.408675 & -5.635554 & ๑. .0०००००० \\
\hline C & 7.238106 & -4.957189 & -1.199567 \\
\hline C & 6.903226 & -3.610514 & -1.196634 \\
\hline $\mathrm{H}$ & -11.708393 & 3.372983 & ๑. . 000000 \\
\hline $\mathrm{H}$ & -9.275164 & 3.323585 & ๑. 000000 \\
\hline $\mathrm{H}$ & -13.051796 & 1.323693 & ๑ . . 000000 \\
\hline $\mathrm{H}$ & -13.051796 & -1.323693 & ๑. . 000000 \\
\hline $\mathrm{H}$ & - 9.275164 & -3.323585 & $\odot . ๑ \odot \odot \odot \odot \odot$ \\
\hline $\mathrm{H}$ & -1.188185 & 3.350302 & 0.000000 \\
\hline $\mathrm{H}$ & 1.188185 & 3.350302 & ๑. . 000000 \\
\hline
\end{tabular}




\begin{tabular}{|c|c|c|c|}
\hline $\mathrm{H}$ & -1.188185 & -3.350302 & 0.000000 \\
\hline $\mathrm{H}$ & 1.188185 & -3.350302 & $\odot . ๑ \odot \odot \odot \odot \odot$ \\
\hline $\mathrm{H}$ & -11.708393 & -3.372983 & ๑. . 000000 \\
\hline $\mathrm{H}$ & 9.275164 & 3.323585 & ๑ . 000000 \\
\hline $\mathrm{H}$ & 11.708393 & 3.372983 & ๑ . . 000000 \\
\hline $\mathrm{H}$ & 13.051796 & 1.323693 & $\odot . \odot \odot \odot \odot \odot \odot$ \\
\hline $\mathrm{H}$ & 9.275164 & -3.323585 & 0.000000 \\
\hline $\mathrm{H}$ & 11.708393 & -3.372983 & 0.000000 \\
\hline $\mathrm{H}$ & 13.051796 & -1.323693 & ๑. . 000000 \\
\hline $\mathrm{H}$ & -6.791307 & 3.083885 & -2.135483 \\
\hline H & -7.373249 & 5.475184 & -2.140820 \\
\hline $\mathrm{H}$ & -7.673802 & 6.685413 & 0.000000 \\
\hline $\mathrm{H}$ & -7.373249 & 5.475184 & 2.140820 \\
\hline $\mathrm{H}$ & -6.791307 & 3.083885 & 2.135483 \\
\hline $\mathrm{H}$ & -3.758227 & 3.085763 & 2.135671 \\
\hline $\mathrm{H}$ & -3.758227 & 3.085763 & -2.135671 \\
\hline $\mathrm{H}$ & -3.149619 & 5.470263 & -2.140687 \\
\hline $\mathrm{H}$ & -2.837458 & 6.677411 & 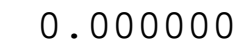 \\
\hline $\mathrm{H}$ & -3.149619 & 5.470263 & 2.140687 \\
\hline H & -6.791307 & -3.083885 & -2.135483 \\
\hline $\mathrm{H}$ & -7.373249 & -5.475184 & -2.140820 \\
\hline $\mathrm{H}$ & -7.673802 & -6.685413 & 0.000000 \\
\hline $\mathrm{H}$ & -7.373249 & -5.475184 & 2.140820 \\
\hline $\mathrm{H}$ & -6.791307 & -3.083885 & 2.135483 \\
\hline $\mathrm{H}$ & -3.758227 & -3.085763 & 2.135671 \\
\hline $\mathrm{H}$ & -3.149619 & -5.470263 & 2.140687 \\
\hline $\mathrm{H}$ & -2.837458 & -6.677411 & ๑. . 000000 \\
\hline $\mathrm{H}$ & -3.149619 & -5.470263 & -2.140687 \\
\hline H & -3.758227 & -3.085763 & -2.135671 \\
\hline $\mathrm{H}$ & 3.758227 & -3.085763 & 2.135671 \\
\hline $\mathrm{H}$ & 3.149619 & -5.470263 & 2.140687 \\
\hline $\mathrm{H}$ & 2.837458 & -6.677411 & ๑. .0००००० \\
\hline $\mathrm{H}$ & 3.149619 & -5.470263 & -2.140687 \\
\hline H & 3.758227 & -3.085763 & -2.135671 \\
\hline $\mathrm{H}$ & 6.791307 & -3.083885 & 2.135483 \\
\hline $\mathrm{H}$ & 7.373249 & -5.475184 & 2.140820 \\
\hline $\mathrm{H}$ & 7.673802 & -6.685413 & 0.000000 \\
\hline $\mathrm{H}$ & 7.373249 & -5.475184 & -2.140820 \\
\hline $\mathrm{H}$ & 6.791307 & -3.083885 & -2.135483 \\
\hline $\mathrm{H}$ & 3.758227 & 3.085763 & -2.135671 \\
\hline $\mathrm{H}$ & 3.149619 & 5.470263 & -2.140687 \\
\hline $\mathrm{H}$ & 2.837458 & 6.677411 & $\odot . ๑ \odot \odot \odot \odot \odot$ \\
\hline H & 3.149619 & 5.470263 & 2.140687 \\
\hline $\mathrm{H}$ & 3.758227 & 3.085763 & 2.135671 \\
\hline $\mathrm{H}$ & 6.791307 & 3.083885 & -2.135483 \\
\hline $\mathrm{H}$ & 7.373249 & 5.475184 & -2.140820 \\
\hline $\mathrm{H}$ & 7.673802 & 6.685413 & $\odot . \odot \odot \odot \odot \odot \odot$ \\
\hline
\end{tabular}




$\begin{array}{rrrr}\mathrm{H} & 7.373249 & 5.475184 & 2.140820 \\ \mathrm{H} & 6.791307 & 3.083885 & 2.135483 \\ & \mathrm{E}=-3740.717141 & \end{array}$

\section{A conformation, electronic ground state, cationic form, B3- $\operatorname{LYP} / \mathrm{SV}(\mathrm{P})$}

\section{( $D_{2}$ symmetry)}

\begin{tabular}{|c|c|c|c|}
\hline c & -2.674610 & 2.644815 & 6.425746 \\
\hline$\tau$ & -1.283670 & 2.633907 & 6.623636 \\
\hline C & -0.646411 & 3.821674 & 7.016390 \\
\hline C & -1.379963 & 4.998755 & 7.183561 \\
\hline C & -2.765134 & 5.001046 & 6.98229 \\
\hline C & -3.411458 & 3.819403 & 6.60649 \\
\hline C & $-\odot .551595$ & 1.329580 & 6.53615 \\
\hline C & -0.189926 & $\odot .702431$ & 5.29157 \\
\hline C & ๑.189926 & -0.702431 & $5.29157=$ \\
\hline C & $\odot .551595$ & -1.329580 & 6.53615 \\
\hline C & ๑. 324776 & $-\odot .641971$ & 7.72913 \\
\hline C & -0.324776 & ๑.641971 & 7.72913 \\
\hline C & $-\odot .185489$ & 1.429270 & 4.037138 \\
\hline C & $-\odot .043659$ & $\odot .725026$ & 2.84823 \\
\hline C & $\odot .043659$ & $-\odot .725026$ & 2.84823 \\
\hline C & ๑.185489 & -1.429270 & 4.037138 \\
\hline C & ๑.584635 & -1.021254 & 9.13255 \\
\hline C & $\odot . ๑ \odot \odot \odot \odot \odot$ & 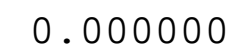 & 9.92764 \\
\hline C & $-\odot .584635$ & 1.021254 & 9.13255 \\
\hline C & ๑.018001 & -1.189010 & 1. 444901 \\
\hline C & $\odot . ๑ \odot \odot \odot \odot \odot$ & $\odot .0 \odot \odot \odot \odot \odot$ & $\odot .68630$ \\
\hline C & -0.018001 & 1.189010 & 1. 44490 \\
\hline C & $\odot . \odot \odot \odot \odot \odot \odot$ & 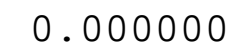 & -0.68630 \\
\hline C & ๑. 018001 & 1.189010 & -1.444901 \\
\hline C & ๑.010893 & 2.391410 & -0.71221 \\
\hline C & -0.010893 & 2.391410 & 0.71221 \\
\hline C & $-\odot .018001$ & -1.189010 & -1.44490 \\
\hline C & -0.010893 & -2.391410 & -0.71221 \\
\hline C & ๑. 010893 & -2.391410 & $\odot .71221$ \\
\hline C & $\odot .043659$ & $\odot .725026$ & -2.84823 \\
\hline C & $-\odot .043659$ & -0.725026 & -2.84823 \\
\hline C & $-\odot .185489$ & -1.429270 & -4.03713 \\
\hline
\end{tabular}




\begin{tabular}{|c|c|c|c|}
\hline C & -0.189926 & -0.702431 & -5.291571 \\
\hline C & ๑. 189926 & 0.702431 & -5.291571 \\
\hline C & ○. 185489 & 1.429270 & -4.037138 \\
\hline C & -0.551595 & -1.329580 & -6.536151 \\
\hline C & -0.324776 & -0.641971 & -7.729138 \\
\hline C & 0.324776 & 0.641971 & -7.729138 \\
\hline C & ๑. 551595 & 1.329580 & -6.536151 \\
\hline C & -0.584635 & -1.021254 & -9.132550 \\
\hline C & 0.000000 & 0.000000 & -9.927640 \\
\hline C & 0.584635 & 1.021254 & -9.132550 \\
\hline C & $\odot .0 \odot \odot \odot \odot \odot$ & $\odot .0 \odot \odot \odot \odot \odot$ & -11.337082 \\
\hline C & 0.660949 & 1.088970 & -11.972294 \\
\hline C & 1.261317 & 2.079571 & -11.205841 \\
\hline C & 1.231619 & 2.066797 & -9.786451 \\
\hline C & -0.660949 & -1.088970 & -11.972294 \\
\hline C & -1.261317 & -2.079571 & -11.205841 \\
\hline C & -1.231619 & -2.066797 & -9.786451 \\
\hline C & 0.000000 & 0.000000 & 11.337082 \\
\hline C & 0.660949 & -1.088970 & 11.972294 \\
\hline C & 1.261317 & -2.079571 & 11.205841 \\
\hline C & 1.231619 & -2.066797 & 9.786451 \\
\hline C & -0.660949 & 1.088970 & 11.972294 \\
\hline C & -1.261317 & 2.079571 & 11.205841 \\
\hline C & -1.231619 & 2.066797 & 9.786451 \\
\hline C & 1.283670 & -2.633907 & 6.623636 \\
\hline C & 2.674610 & -2.644815 & 6.425746 \\
\hline C & 3.411458 & -3.819403 & 6.606496 \\
\hline C & 2.765134 & -5.001046 & 6.982292 \\
\hline C & 1.379963 & -4.998755 & 7.183561 \\
\hline C & ๑. 646411 & -3.821674 & 7.016390 \\
\hline C & -0.180509 & 2.924799 & 3.938261 \\
\hline C & -1.314149 & 3.649168 & 3.537590 \\
\hline C & -1.243102 & 5.032208 & 3.352949 \\
\hline C & -0.032767 & 5.708109 & 3.545215 \\
\hline C & 1.105803 & 4.992642 & 3.928766 \\
\hline C & 1.031392 & 3.610494 & 4.126121 \\
\hline C & ๑. 180509 & -2.924799 & 3.938261 \\
\hline C & -1.031392 & -3.610494 & 4.126121 \\
\hline C & -1.105803 & -4.992642 & 3.928766 \\
\hline C & 0.032767 & -5.708109 & 3.545215 \\
\hline C & 1.243102 & -5.032208 & 3. 352949 \\
\hline C & 1.314149 & -3.649168 & 3.537590 \\
\hline C & ๑. 180509 & 2.924799 & -3.938261 \\
\hline C & -1.031392 & 3.610494 & -4.126121 \\
\hline C & -1.105803 & 4.992642 & -3.928766 \\
\hline C & 0.032767 & 5.708109 & -3.545215 \\
\hline C & 1.243102 & 5.032208 & -3.35294 \\
\hline
\end{tabular}




\begin{tabular}{|c|c|c|c|}
\hline C & 1.314149 & 3.649168 & -3.537590 \\
\hline C & -0.180509 & -2.924799 & -3.938261 \\
\hline C & $-1 \cdot 314149$ & -3.649168 & -3.537590 \\
\hline C & -1.243102 & -5.032208 & -3.352949 \\
\hline C & -0.032767 & -5.708109 & -3.545215 \\
\hline C & 1.105803 & -4.992642 & -3.928766 \\
\hline C & 1.031392 & -3.610494 & -4.126121 \\
\hline C & 1.283670 & 2.633907 & -6.623636 \\
\hline C & ๑. 646411 & 3.821674 & -7.01639 \\
\hline C & 1.379963 & 4.998755 & -7.183561 \\
\hline C & 2.765134 & 5.001046 & -6.982292 \\
\hline C & 3.411458 & 3.819403 & -6.606496 \\
\hline C & 2.674610 & 2.644815 & -6.425746 \\
\hline C & -1.283670 & -2.633907 & -6.62363 \\
\hline C & -2.674610 & -2.644815 & -6.425746 \\
\hline C & -3.411458 & -3.819403 & -6.606496 \\
\hline C & -2.765134 & -5.001046 & -6.9822 \\
\hline C & -1.379963 & -4.998755 & -7.183561 \\
\hline C & -0.646411 & -3.821674 & - 7.016390 \\
\hline $\mathrm{H}$ & -1.773877 & -2.905564 & -11.707819 \\
\hline $\mathrm{H}$ & -1.719571 & -2.874772 & - 9.241913 \\
\hline $\mathrm{H}$ & -0.698071 & -1.140714 & -13.064856 \\
\hline $\mathrm{H}$ & ๑. 698071 & 1.140714 & -13.064856 \\
\hline $\mathrm{H}$ & 1.719571 & 2.874772 & -9.241913 \\
\hline $\mathrm{H}$ & -0.018541 & -3.357811 & -1.217391 \\
\hline $\mathrm{H}$ & 0.018541 & -3.357811 & 1.217391 \\
\hline $\mathrm{H}$ & ๑ . 018541 & 3.357811 & -1.217391 \\
\hline $\mathrm{H}$ & -0.018541 & 3.357811 & 1.217391 \\
\hline $\mathrm{H}$ & 1.773877 & 2.905564 & -11.707819 \\
\hline $\mathrm{H}$ & 1.719571 & -2.874772 & 9.241913 \\
\hline $\mathrm{H}$ & 1.773877 & -2.905564 & 11.707819 \\
\hline $\mathrm{H}$ & ๑. 698071 & -1.140714 & 13.064856 \\
\hline $\mathrm{H}$ & -1.719571 & 2.874772 & 9.241913 \\
\hline $\mathrm{H}$ & -1.773877 & 2.905564 & 11.707819 \\
\hline $\mathrm{H}$ & -0.698071 & 1.140714 & 13.064856 \\
\hline $\mathrm{H}$ & -3.185140 & -1.720173 & -6.139718 \\
\hline $\mathrm{H}$ & -4.495467 & -3.809299 & -6.456089 \\
\hline $\mathrm{H}$ & -3.339519 & -5.921880 & -7.122300 \\
\hline $\mathrm{H}$ & -0.867009 & -5.919127 & -7.478646 \\
\hline $\mathrm{H}$ & ๑. 432958 & -3.823508 & -7.189717 \\
\hline $\mathrm{H}$ & -2.259722 & -3.125466 & -3.375009 \\
\hline $\mathrm{H}$ & 1.925769 & -3.053085 & -4.42049 \\
\hline $\mathrm{H}$ & 2.058385 & -5.511381 & -4.074548 \\
\hline $\mathrm{H}$ & ๑ . 022150 & -6.790708 & -3.395441 \\
\hline $\mathrm{H}$ & -2.138841 & -5.584989 & -3.05389 \\
\hline $\mathrm{H}$ & -0.432958 & 3.823508 & -7.18971 \\
\hline $\mathrm{H}$ & 0.867009 & 5.919127 & -7.47864 \\
\hline
\end{tabular}




\begin{tabular}{rrrr}
$\mathrm{H}$ & 3.339519 & 5.921880 & -7.122300 \\
$\mathrm{H}$ & 4.495467 & 3.809299 & -6.456089 \\
$\mathrm{H}$ & 3.185140 & 1.720173 & -6.139718 \\
$\mathrm{H}$ & -1.925769 & 3.053085 & -4.420491 \\
$\mathrm{H}$ & -2.058385 & 5.511381 & -4.074548 \\
$\mathrm{H}$ & -0.022150 & 6.790708 & -3.395441 \\
$\mathrm{H}$ & 2.138841 & 5.584989 & -3.053895 \\
$\mathrm{H}$ & 2.259722 & 3.125466 & -3.375009 \\
$\mathrm{H}$ & -2.259722 & 3.125466 & 3.375009 \\
$\mathrm{H}$ & -2.138841 & 5.584989 & 3.053895 \\
$\mathrm{H}$ & 0.022150 & 6.790708 & 3.395441 \\
$\mathrm{H}$ & 2.058385 & 5.511381 & 4.074548 \\
$\mathrm{H}$ & 1.925769 & 3.053085 & 4.420491 \\
$\mathrm{H}$ & 0.432958 & 3.823508 & 7.189717 \\
$\mathrm{H}$ & -0.867009 & 5.919127 & 7.478646 \\
$\mathrm{H}$ & -3.339519 & 5.921880 & 7.122300 \\
$\mathrm{H}$ & -4.495467 & 3.809299 & 6.456089 \\
$\mathrm{H}$ & -3.185140 & 1.720173 & 6.139718 \\
$\mathrm{H}$ & -1.925769 & -3.053085 & 4.420491 \\
$\mathrm{H}$ & -2.058385 & -5.511381 & 4.074548 \\
$\mathrm{H}$ & -0.022150 & -6.790708 & 3.395441 \\
$\mathrm{H}$ & 2.138841 & -5.584989 & 3.053895 \\
$\mathrm{H}$ & 2.259722 & -3.125466 & 3.375009 \\
$\mathrm{H}$ & -0.432958 & -3.823508 & 7.189717 \\
$\mathrm{H}$ & 0.867009 & -5.919127 & 7.478646 \\
$\mathrm{H}$ & 3.339519 & -5.921880 & 7.122300 \\
$\mathrm{H}$ & 4.495467 & -3.809299 & 6.456089 \\
$\mathrm{H}$ & 3.185140 & -1.720173 & 6.139718 \\
& $\mathrm{E}=-3765.208852$ & \\
\hline & & &
\end{tabular}

A conformation, electronic ground state, anionic form, B3LYP/SV (P)

( $D_{2}$ symmetry)

$\begin{array}{llll}C & -2.687234 & 2.628141 & 6.458106 \\ C & -1.293543 & 2.625662 & 6.636881 \\ C & -0.667388 & 3.824685 & 7.012451 \\ C & -1.408588 & 4.996297 & 7.185153\end{array}$




\begin{tabular}{|c|c|c|c|}
\hline $\mathrm{C}$ & -2.795936 & 4.986670 & 7.002334 \\
\hline $\mathrm{C}$ & -3.433934 & 3.796157 & 6.640123 \\
\hline $\mathrm{C}$ & -0.549997 & 1.325830 & 6.547108 \\
\hline $\mathrm{C}$ & $-\odot .18510 \odot$ & 0.708546 & 5.290811 \\
\hline $\mathrm{C}$ & 0.185100 & -0.708546 & 5.290811 \\
\hline $\mathrm{C}$ & 0.549997 & -1.325830 & 6.547108 \\
\hline C & ๑. 329076 & -0.646203 & 7.735697 \\
\hline C & -0.329076 & 0.646203 & 7.735697 \\
\hline C & -0.188500 & 1.425719 & 4.042363 \\
\hline C & -0.042844 & $\odot .733392$ & 2.843699 \\
\hline C & ๑ . 042844 & -0.733392 & 2.843699 \\
\hline C & 0.188500 & -1.425719 & 4.042363 \\
\hline C & 0.593069 & -1.019353 & 9.141019 \\
\hline C & ๑ . . 000000 & ๑ . . 000000 & 9.939738 \\
\hline C & -0.593069 & 1.019353 & 9.141019 \\
\hline C & ๑ . 016723 & -1.192536 & 1.449093 \\
\hline C & 0.000000 & 0.000000 & $\odot .689549$ \\
\hline C & -0.016723 & 1.192536 & 1.449093 \\
\hline C & ๑ . 000000 & ๑ . $00000 \odot$ & -0.689549 \\
\hline C & ๑ . 016723 & 1.192536 & -1.449093 \\
\hline C & 0.010320 & 2.399155 & -0.708159 \\
\hline C & -0.010320 & 2.399155 & $\odot .708159$ \\
\hline C & -0.016723 & -1.192536 & -1.449093 \\
\hline C & -0.010320 & -2.399155 & -0.708159 \\
\hline C & ๑ . 010320 & - 2. 399155 & $\odot .708159$ \\
\hline C & 0.042844 & 0.733392 & -2.843699 \\
\hline C & -0.042844 & -0.733392 & -2.843699 \\
\hline C & -0.188500 & -1.425719 & -4.042363 \\
\hline C & -0.185100 & -0.708546 & -5.290811 \\
\hline C & ๑ . 185100 & 0.708546 & -5.290811 \\
\hline C & 0.188500 & 1.425719 & -4.042363 \\
\hline C & -0.549997 & -1.325830 & -6.547108 \\
\hline C & -0.329076 & -0.646203 & -7.735697 \\
\hline C & $\odot .329076$ & 0.646203 & -7.735697 \\
\hline C & 0.549997 & 1.325830 & -6.547108 \\
\hline C & -0.593069 & -1.019353 & -9.141019 \\
\hline C & 0.000000 & 0.000000 & -9.939738 \\
\hline C & 0.593069 & 1.019353 & -9.141019 \\
\hline C & ๑. . $000 \odot \odot ०$ & $\odot .0 \odot ० ० \odot \odot$ & -11.351971 \\
\hline C & 0.668056 & 1.085851 & -11.988164 \\
\hline C & 1.272706 & 2.070805 & -11.219106 \\
\hline C & 1.245298 & 2.059854 & -9.799098 \\
\hline C & $-\odot .668056$ & -1.085851 & -11.988164 \\
\hline C & -1.272706 & -2.070805 & -11.219106 \\
\hline C & -1.245298 & -2.059854 & -9.799098 \\
\hline C & 0.000000 & 0.000000 & 11.351971 \\
\hline C & $\odot .668056$ & -1.085851 & 11.988164 \\
\hline
\end{tabular}




\begin{tabular}{|c|c|c|c|}
\hline C & 1.272706 & -2.070805 & 11.219106 \\
\hline C & 1.245298 & -2.059854 & 9.799098 \\
\hline C & -0.668056 & 1.085851 & 11.988164 \\
\hline C & -1.272706 & 2.070805 & 11.219106 \\
\hline C & -1.245298 & 2.059854 & 9.799098 \\
\hline C & 1.293543 & -2.625662 & 6.636881 \\
\hline C & 2.687234 & -2.628141 & 6.458106 \\
\hline C & 3.433934 & -3.796157 & 6.640123 \\
\hline C & 2.795936 & -4.986670 & 7.002334 \\
\hline C & 1.408588 & -4.996297 & 7.185153 \\
\hline $\mathrm{C}$ & $\odot .667388$ & -3.824685 & 7.012451 \\
\hline C & -0.206244 & 2.922843 & 3.943478 \\
\hline C & -1.348969 & 3.634316 & 3.544891 \\
\hline C & -1.304077 & 5.018556 & 3.361548 \\
\hline C & -0.107167 & 5.717208 & 3.555029 \\
\hline C & 1.043068 & 5.019023 & 3.935715 \\
\hline $\mathrm{C}$ & 0. 990984 & 3.635106 & 4.128084 \\
\hline C & ๑. 206244 & -2.922843 & 3.943478 \\
\hline C & -0.990984 & -3.635106 & 4.128084 \\
\hline $\mathrm{C}$ & -1.043068 & -5.019023 & 3.935715 \\
\hline $\mathrm{C}$ & 0.107167 & -5.717208 & 3.555029 \\
\hline C & 1.304077 & -5.018556 & 3.361548 \\
\hline C & 1.348969 & -3.634316 & 3.544891 \\
\hline C & ○. 206244 & 2.922843 & -3.943478 \\
\hline $\mathrm{C}$ & -0.990984 & 3.635106 & -4.128084 \\
\hline $\mathrm{C}$ & -1.043068 & 5.019023 & -3.935715 \\
\hline C & 0.107167 & 5.717208 & -3.555029 \\
\hline C & 1.304077 & 5.018556 & -3.361548 \\
\hline $\mathrm{C}$ & 1.348969 & 3.634316 & -3.544891 \\
\hline C & -0.206244 & -2.922843 & -3.943478 \\
\hline $\mathrm{C}$ & -1.348969 & -3.634316 & -3.544891 \\
\hline C & -1.304077 & -5.018556 & -3.361548 \\
\hline C & -0.107167 & -5.717208 & -3.555029 \\
\hline $\mathrm{C}$ & 1.043068 & -5.019023 & -3.935715 \\
\hline $\mathrm{C}$ & ○. 990984 & -3.635106 & -4.128084 \\
\hline C & 1.293543 & 2.625662 & -6.636881 \\
\hline C & 0.667388 & 3.824685 & -7.012451 \\
\hline C & 1.408588 & 4.996297 & -7.185153 \\
\hline C & 2.795936 & 4.986670 & -7.002334 \\
\hline C & 3.433934 & 3.796157 & -6.640123 \\
\hline C & 2.687234 & 2.628141 & -6.458106 \\
\hline $\mathrm{C}$ & -1.293543 & -2.625662 & -6.636881 \\
\hline $\mathrm{C}$ & -2.687234 & -2.628141 & -6.458106 \\
\hline C & -3.433934 & -3.796157 & -6.640123 \\
\hline C & -2.795936 & -4.986670 & -7.002334 \\
\hline C & -1.408588 & -4.996297 & -7.185153 \\
\hline C & -0.667388 & -3.824685 & -7.012451 \\
\hline
\end{tabular}




\begin{tabular}{|c|c|c|c|}
\hline $\mathrm{H}$ & -1.790365 & -2.895965 & -11.720642 \\
\hline $\mathrm{H}$ & -1.738101 & -2.866058 & -9.255987 \\
\hline $\mathrm{H}$ & -0.704526 & -1.136221 & -13.081768 \\
\hline $\mathrm{H}$ & 0.704526 & 1.136221 & -13.081768 \\
\hline $\mathrm{H}$ & 1.738101 & 2.866058 & -9.255987 \\
\hline $\mathrm{H}$ & -0.015502 & -3.366979 & -1.212209 \\
\hline $\mathrm{H}$ & 0. . 015502 & -3.366979 & 1.212209 \\
\hline $\mathrm{H}$ & 0.015502 & 3.366979 & -1.212209 \\
\hline $\mathrm{H}$ & -0.015502 & 3.366979 & 1. 212209 \\
\hline $\mathrm{H}$ & 1.790365 & 2.895965 & -11.720642 \\
\hline $\mathrm{H}$ & 1.738101 & -2.866058 & 9.255987 \\
\hline $\mathrm{H}$ & 1.790365 & -2.895965 & 11.720642 \\
\hline $\mathrm{H}$ & 0.704526 & -1.136221 & 13.081768 \\
\hline $\mathrm{H}$ & -1.738101 & 2.866058 & 9.255987 \\
\hline $\mathrm{H}$ & -1.790365 & 2.895965 & 11.720642 \\
\hline $\mathrm{H}$ & -0.704526 & 1.136221 & 13.081768 \\
\hline $\mathrm{H}$ & -3.188656 & -1.696478 & -6.179335 \\
\hline $\mathrm{H}$ & -4.519720 & -3.775101 & -6.498427 \\
\hline $\mathrm{H}$ & -3.377013 & -5.904386 & -7.141309 \\
\hline $\mathrm{H}$ & -0.898616 & -5.923545 & -7.465912 \\
\hline $\mathrm{H}$ & 0.414319 & -3.835589 & -7.168825 \\
\hline $\mathrm{H}$ & -2.282008 & -3.091116 & -3.374310 \\
\hline $\mathrm{H}$ & 1.894216 & -3.088641 & -4.416012 \\
\hline $\mathrm{H}$ & 1.988461 & -5.553219 & -4.079001 \\
\hline $\mathrm{H}$ & -0.070751 & -6.801228 & -3.403922 \\
\hline $\mathrm{H}$ & -2.209166 & -5.554597 & -3.057868 \\
\hline $\mathrm{H}$ & -0.414319 & 3.835589 & -7.168825 \\
\hline $\mathrm{H}$ & ๑. 898616 & 5.923545 & -7.465912 \\
\hline $\mathrm{H}$ & 3.377013 & 5.904386 & -7.141309 \\
\hline $\mathrm{H}$ & 4.519720 & 3.775101 & -6.498427 \\
\hline $\mathrm{H}$ & 3.188656 & 1.696478 & -6.179335 \\
\hline $\mathrm{H}$ & -1.894216 & 3.088641 & -4.416012 \\
\hline $\mathrm{H}$ & -1.988461 & 5.553219 & -4.079001 \\
\hline $\mathrm{H}$ & ๑. . 070751 & 6.801228 & -3.403922 \\
\hline $\mathrm{H}$ & 2.209166 & 5.554597 & -3.057868 \\
\hline $\mathrm{H}$ & 2.282008 & 3.091116 & -3.374310 \\
\hline $\mathrm{H}$ & -2.282008 & 3.091116 & 3.374310 \\
\hline $\mathrm{H}$ & -2.209166 & 5.554597 & 3.057868 \\
\hline $\mathrm{H}$ & $-\odot .070751$ & 6.801228 & 3.403922 \\
\hline $\mathrm{H}$ & 1.988461 & 5.553219 & 4.079001 \\
\hline $\mathrm{H}$ & 1.894216 & 3.088641 & 4.416012 \\
\hline $\mathrm{H}$ & 0. 414319 & 3.835589 & 7.168825 \\
\hline $\mathrm{H}$ & -0.898616 & 5.923545 & 7.465912 \\
\hline $\mathrm{H}$ & -3.377013 & 5.904386 & 7.141309 \\
\hline $\mathrm{H}$ & -4.519720 & 3.775101 & 6.498427 \\
\hline $\mathrm{H}$ & -3.188656 & 1.696478 & 6.179335 \\
\hline $\mathrm{H}$ & -1.894216 & -3.088641 & 4.416012 \\
\hline
\end{tabular}




$\begin{array}{lrrr}\mathrm{H} & -1.988461 & -5.553219 & 4.079001 \\ \mathrm{H} & 0.070751 & -6.801228 & 3.403922 \\ \mathrm{H} & 2.209166 & -5.554597 & 3.057868 \\ \mathrm{H} & 2.282008 & -3.091116 & 3.374310 \\ \mathrm{H} & -0.414319 & -3.835589 & 7.168825 \\ \mathrm{H} & 0.898616 & -5.923545 & 7.465912 \\ \mathrm{H} & 3.377013 & -5.904386 & 7.141309 \\ \mathrm{H} & 4.519720 & -3.775101 & 6.498427 \\ \mathrm{H} & 3.188656 & -1.696478 & 6.179335\end{array}$

B conformation, electronic ground state, neutral form, B3LYP/SV (P)

\section{( $D_{2}$ symmetry)}


$\begin{array}{llll}\text { C } & -6.502381 & -3.274087 & -1.848423\end{array}$

\begin{tabular}{|c|c|c|c|}
\hline$c$ & -6.646513 & -2.883068 & -0.506694 \\
\hline & -7.000633 & -3.856079 & $\odot .441393$ \\
\hline C & -7.183994 & -5.188223 & 0.06275 \\
\hline C & -7.033553 & -5.567590 & -1.27595 \\
\hline & -6.694474 & -4.605034 & -2.23181 \\
\hline & -6.550248 & -1.426630 & -0.16011 \\
\hline c & -5.297394 & -0.726624 & 0.01087 \\
\hline C & -5.297170 & $\odot .728245$ & -0.00224 \\
\hline & -6.549483 & 1.428691 & 0.1707 \\
\hline & -7.735628 & 0.710255 & 0.1393 \\
\hline$C$ & -7.736083 & -0.707759 & -0.1268 \\
\hline C & -4.041332 & 1.426559 & -0.1877 \\
\hline C & -2.856357 & 0.713214 & -0.1418 \\
\hline C & -2.856348 & -0.712305 & 0.1465 \\
\hline C & -4.041459 & -1.425314 & 0.1943 \\
\hline c & -1.444530 & -1.155694 & 0.2896 \\
\hline C & -0.686724 & $\odot .00 \odot 107$ & ๑.00०5 \\
\hline c & -1.444639 & 1.156152 & -0.2872 \\
\hline c & -0.717116 & -2.307196 & 0.6064 \\
\hline C & $\odot .717390$ & -2.307427 & 0.6052 \\
\hline c & 1.444639 & -1.156152 & 0.2872 \\
\hline C & $\odot .686724$ & -0.000107 & -0.0005 \\
\hline C & 1.444530 & 1.155694 & -0.2896 \\
\hline C & 2.856348 & 0.712305 & -0.1465 \\
\hline C & 2.856357 & -0.713214 & 0.1418 \\
\hline c & 4.041332 & -1.426559 & 0.1877 \\
\hline C & 5.297170 & -0.728245 & 0.0022 \\
\hline C & 5.297394 & 0.726624 & -0.0108 \\
\hline C & 4.041459 & 1.425314 & -0.1943 \\
\hline c & 6.549483 & -1.428691 & -0.1707 \\
\hline c & 7.735628 & -0.710255 & -0.1393 \\
\hline C & 7.736083 & $\odot .707759$ & 0.1268 \\
\hline C & 6.550248 & 1.426630 & 0.1601 \\
\hline c & 9.146382 & 1.143618 & 0.2690 \\
\hline C & 9.942062 & -0.001643 & $-\odot .0079$ \\
\hline C & 9.145543 & -1.146622 & -0.2837 \\
\hline C & 11.352371 & $-\odot .001891$ & $-\odot .0091$ \\
\hline c & 11.987675 & -1.233812 & -0.3376 \\
\hline c & 11.220721 & -2.349881 & -0.6330 \\
\hline c & 9.797902 & -2.328550 & -0.6086 \\
\hline c & 11.988639 & 1.229808 & 0.3182 \\
\hline c & 11.222552 & 2.346140 & 0.6149 \\
\hline C & 9.799689 & 2.325307 & 0.5929 \\
\hline C & -0.717390 & 2.307427 & -0.6052 \\
\hline , & 0.717116 & 2.307196 & -0.6064 \\
\hline & -9.145543 & 1.146622 & 0.2837 \\
\hline
\end{tabular}




\begin{tabular}{|c|c|c|c|}
\hline C & -9.942062 & ๑. 001643 & ๑. . 007997 \\
\hline C & -9.146382 & -1.143618 & -0.269051 \\
\hline C & -11.352371 & ๑ . 001891 & ๑ . 009144 \\
\hline C & -11.988639 & -1.229808 & -0.318278 \\
\hline C & -11.222552 & -2.346140 & -0.614959 \\
\hline C & -9.799689 & -2.325307 & -0.592930 \\
\hline C & -11.987675 & 1.233812 & $\odot .337606$ \\
\hline C & -11.220721 & 2.349881 & 0.633028 \\
\hline C & -9.797902 & 2.328550 & 0.608696 \\
\hline C & 6.646513 & 2.883068 & 0.506694 \\
\hline C & 6.502381 & 3.274087 & 1.848423 \\
\hline C & 6.694474 & 4.605034 & 2.231814 \\
\hline C & 7.033553 & 5.567590 & 1. 275956 \\
\hline C & 7.183994 & 5.188223 & -0.062751 \\
\hline C & 7.000633 & 3.856079 & -0.441393 \\
\hline C & 6.644672 & -2.885146 & -0.517575 \\
\hline C & 6.498392 & -3.276050 & -1.859112 \\
\hline C & 6.689395 & -4.607051 & -2.242845 \\
\hline C & 7.029521 & -5.569787 & -1.287531 \\
\hline C & 7.182093 & -5.190540 & ๑. . 050965 \\
\hline C & 6.999817 & -3.858340 & 0. 429936 \\
\hline C & 3.933846 & 2.872898 & -0.574636 \\
\hline C & 4.075329 & 3.234707 & -1.924942 \\
\hline C & 3.868879 & 4.554138 & -2.339006 \\
\hline C & 3.516631 & 5.534407 & -1.406156 \\
\hline C & 3.366380 & 5.183706 & -0.059790 \\
\hline C & 3.564503 & 3.862772 & 0.349729 \\
\hline C & 3.933956 & -2.874101 & 0.568259 \\
\hline C & 4.077416 & -3.235888 & 1.918367 \\
\hline C & 3.871356 & -4.555267 & 2.332788 \\
\hline C & 3.517540 & -5.535509 & 1.400502 \\
\hline C & 3.365340 & -5.184833 & ๑. 054349 \\
\hline C & 3.563074 & -3.863947 & -0.355512 \\
\hline C & -3.933846 & -2.872898 & 0.574636 \\
\hline C & -4.075329 & -3.234707 & 1.924942 \\
\hline C & -3.868879 & -4.554138 & 2.339006 \\
\hline C & -3.516631 & -5.534407 & 1.406156 \\
\hline C & -3.366380 & -5.183706 & ๑. . 059790 \\
\hline C & -3.564503 & -3.862772 & -0.349729 \\
\hline C & -3.933956 & 2.874101 & -0.568259 \\
\hline C & -4.077416 & 3.235888 & -1.918367 \\
\hline C & -3.871356 & 4.555267 & -2.332788 \\
\hline C & -3.517540 & 5.535509 & -1.400502 \\
\hline C & -3.365340 & 5.184833 & -0.054349 \\
\hline C & -3.563074 & 3.863947 & ๑. 355512 \\
\hline C & -6.644672 & 2.885146 & 0.517575 \\
\hline C & -6.999817 & 3.858340 & -0.429936 \\
\hline
\end{tabular}




\begin{tabular}{|c|c|c|c|}
\hline $\mathrm{C}$ & -7.182093 & 5.190540 & -0.050965 \\
\hline $\mathrm{C}$ & -7.029521 & 5.569787 & 1.287531 \\
\hline $\mathrm{C}$ & -6.689395 & 4.607051 & 2.242845 \\
\hline $\mathrm{C}$ & -6.498392 & 3.276050 & 1.859112 \\
\hline $\mathrm{H}$ & -11.723222 & -3.284991 & -0.872428 \\
\hline $\mathrm{H}$ & -9.256257 & -3.238947 & -0.832833 \\
\hline $\mathrm{H}$ & -13.081729 & -1.288677 & -0.337778 \\
\hline $\mathrm{H}$ & -13.080711 & 1.293055 & ๑. 358884 \\
\hline $\mathrm{H}$ & -9.253756 & 3.242005 & 0.847674 \\
\hline $\mathrm{H}$ & -1.218783 & -3.241418 & ๑. 862354 \\
\hline $\mathrm{H}$ & 1.219190 & -3.241811 & 0.860290 \\
\hline $\mathrm{H}$ & -1.219190 & 3.241811 & -0.860290 \\
\hline $\mathrm{H}$ & 1.218783 & 3.241418 & -0.862354 \\
\hline $\mathrm{H}$ & -11.720639 & 3.288913 & 0.891289 \\
\hline $\mathrm{H}$ & 9.253756 & -3.242005 & -0.847674 \\
\hline $\mathrm{H}$ & 11.720639 & -3.288913 & -0.891289 \\
\hline $\mathrm{H}$ & 13.080711 & -1.293055 & -0.358884 \\
\hline $\mathrm{H}$ & 9.256257 & 3.238947 & 0.832833 \\
\hline $\mathrm{H}$ & 11.723222 & 3.284991 & 0.872428 \\
\hline $\mathrm{H}$ & 13.081729 & 1.288677 & 0.337778 \\
\hline $\mathrm{H}$ & -7.131753 & -3.563358 & 1.486387 \\
\hline $\mathrm{H}$ & -7.449553 & -5.934514 & $\odot .817996$ \\
\hline $\mathrm{H}$ & -7.182321 & -6.610662 & -1.572796 \\
\hline $\mathrm{H}$ & -6.581675 & -4.889847 & -3.282892 \\
\hline $\mathrm{H}$ & -6.245788 & -2.521607 & -2.600252 \\
\hline $\mathrm{H}$ & -4.341883 & -2.468182 & 2.658915 \\
\hline $\mathrm{H}$ & -3.433625 & -3.592378 & -1.400768 \\
\hline $\mathrm{H}$ & -3.088704 & -5.943526 & -0.677431 \\
\hline $\mathrm{H}$ & -3.356041 & -6.568507 & 1.727226 \\
\hline $\mathrm{H}$ & -3.979826 & -4.815825 & 3.396273 \\
\hline $\mathrm{H}$ & -7.132573 & 3.565725 & -1.474752 \\
\hline $\mathrm{H}$ & -7.448478 & 5.936968 & -0.805785 \\
\hline $\mathrm{H}$ & -7.177458 & 6.612900 & 1.584641 \\
\hline $\mathrm{H}$ & -6.574928 & 4.891774 & 3.293767 \\
\hline $\mathrm{H}$ & -6.240968 & 2.523436 & 2.610523 \\
\hline $\mathrm{H}$ & -4.345236 & 2.469392 & -2.651899 \\
\hline $\mathrm{H}$ & -3.983868 & 4.816934 & -3.389893 \\
\hline $\mathrm{H}$ & -3.357254 & 6.569569 & -1.721854 \\
\hline $\mathrm{H}$ & -3.086413 & 5.944634 & 0.682424 \\
\hline $\mathrm{H}$ & -3.430669 & 3.593573 & 1.406363 \\
\hline $\mathrm{H}$ & 4.341883 & 2.468182 & -2.658915 \\
\hline $\mathrm{H}$ & 3.979826 & 4.815825 & -3.396273 \\
\hline $\mathrm{H}$ & 3.356041 & 6.568507 & -1.727226 \\
\hline $\mathrm{H}$ & 3.088704 & 5.943526 & 0.677431 \\
\hline $\mathrm{H}$ & 3.433625 & 3.592378 & 1.400768 \\
\hline $\mathrm{H}$ & 7.131753 & 3.563358 & -1.486387 \\
\hline $\mathrm{H}$ & 7.449553 & 5.934514 & -0.817996 \\
\hline
\end{tabular}




$\begin{array}{rrrr}\mathrm{H} & 7.182321 & 6.610662 & 1.572796 \\ \mathrm{H} & 6.581675 & 4.889847 & 3.282892 \\ \mathrm{H} & 6.245788 & 2.521607 & 2.600252 \\ \mathrm{H} & 4.345236 & -2.469392 & 2.651899 \\ \mathrm{H} & 3.983868 & -4.816934 & 3.389893 \\ \mathrm{H} & 3.357254 & -6.569569 & 1.721854 \\ \mathrm{H} & 3.086413 & -5.944634 & -0.682424 \\ \mathrm{H} & 3.430669 & -3.593573 & -1.406363 \\ \mathrm{H} & 7.132573 & -3.565725 & 1.474752 \\ \mathrm{H} & 7.448478 & -5.936968 & 0.805785 \\ \mathrm{H} & 7.177458 & -6.612900 & -1.584641 \\ \mathrm{H} & 6.574928 & -4.891774 & -3.293767 \\ \mathrm{H} & 6.240968 & -2.523436 & -2.610523 \\ & \mathrm{E}=-3765.423707 & \end{array}$

B conformation, electronic ground state, cationic form, B3LYP/SV (P)

( $D_{2}$ symmetry)

$\begin{array}{cccc}C & -6.456603 & -3.262904 & -1.867409 \\ C & -6.629892 & -2.880699 & -0.526510 \\ C & -7.007434 & -3.854506 & 0.411754 \\ C & -7.183542 & -5.184545 & 0.022619 \\ C & -7.005942 & -5.557133 & -1.314824 \\ C & -6.645349 & -4.591691 & -2.259879 \\ C & -6.536988 & -1.427722 & -0.171526 \\ C & -5.291764 & -0.727055 & 0.006953 \\ C & -5.291536 & 0.728652 & 0.001616 \\ C & -6.536255 & 1.429730 & 0.181989 \\ C & -7.729326 & 0.706880 & 0.145933 \\ C & -7.729767 & -0.704448 & -0.133823 \\ C & -4.036594 & 1.428273 & -0.193780 \\ C & -2.848032 & 0.711401 & -0.145874\end{array}$




\begin{tabular}{|c|c|c|c|}
\hline C & -2.848023 & -0.710480 & 0.150718 \\
\hline C & -4.036716 & -1.427028 & 0.200423 \\
\hline C & -1.444793 & -1.152253 & ○. 294355 \\
\hline C & -0.686257 & 0.000120 & 0.000632 \\
\hline C & -1.444909 & 1.152737 & -0.291802 \\
\hline C & -0.712186 & -2.309953 & 0.619673 \\
\hline C & ๑. 712491 & -2.310186 & ๑. 618429 \\
\hline C & 1.444909 & -1.152737 & ๑. 291802 \\
\hline C & ๑. 686257 & -0.000120 & -0.000632 \\
\hline C & 1.444793 & 1.152253 & -0.294355 \\
\hline C & 2.848023 & $\odot .710480$ & -0.150718 \\
\hline C & 2.848032 & -0.711401 & 0.145874 \\
\hline C & 4.036594 & -1.428273 & 0.193780 \\
\hline C & 5.291536 & -0.728652 & -0.001616 \\
\hline C & 5.291764 & $\odot .727055$ & -0.006953 \\
\hline C & 4.036716 & 1.427028 & -0.200423 \\
\hline C & 6.536255 & -1.429730 & -0.181989 \\
\hline $\mathrm{C}$ & 7.729326 & -0.706880 & -0.145933 \\
\hline C & 7.729767 & 0.704448 & 0.133823 \\
\hline C & 6.536988 & 1.427722 & $\odot .171526$ \\
\hline C & 9.133425 & 1.140027 & 0.278772 \\
\hline C & 9.928124 & -0.001628 & -0.007613 \\
\hline C & 9.132600 & -1.142973 & -0.292917 \\
\hline C & 11.337584 & -0.001894 & -0.008635 \\
\hline $\mathrm{C}$ & 11.972346 & -1.229761 & -0.348635 \\
\hline C & 11.205467 & -2.346213 & -0.656307 \\
\hline C & 9.786091 & -2.325318 & -0.631056 \\
\hline C & 11.973304 & 1.225736 & 0.330430 \\
\hline C & 11.207282 & 2.342475 & ๑. 639192 \\
\hline C & 9.787867 & 2.322121 & ๑. 615967 \\
\hline C & -0.712491 & 2.310186 & -0.618429 \\
\hline C & ๑. 712186 & 2.309953 & -0.619673 \\
\hline C & -9.132600 & 1.142973 & ○. 292917 \\
\hline C & -9.928124 & ๑ . 001628 & 0.007613 \\
\hline C & -9.133425 & -1.140027 & -0.278772 \\
\hline C & -11.337584 & ○. .001894 & ๑. .008635 \\
\hline C & -11.973304 & -1.225736 & -0.330430 \\
\hline C & -11.207282 & -2.342475 & -0.639192 \\
\hline C & -9.787867 & -2.322121 & -0.615967 \\
\hline C & -11.972346 & 1. 229761 & 0.348635 \\
\hline C & -11.205467 & 2.346213 & 0.656307 \\
\hline C & -9.786091 & 2.325318 & 0.631056 \\
\hline C & 6.629892 & 2.880699 & 0.526510 \\
\hline C & 6.456603 & 3.262904 & 1.867409 \\
\hline C & 6.645349 & 4.591691 & 2.259879 \\
\hline C & 7.005942 & 5.557133 & 1.314824 \\
\hline C & 7.183542 & 5.184545 & -0.022619 \\
\hline
\end{tabular}




\begin{tabular}{|c|c|c|c|}
\hline C & 7.007434 & 3.854506 & -0.411754 \\
\hline C & 6.628112 & -2.882698 & -0.537280 \\
\hline C & 6.452447 & -3.264708 & -1.877923 \\
\hline C & 6.640098 & -4.593518 & -2.270850 \\
\hline C & 7.001972 & -5.559177 & -1.326511 \\
\hline C & 7.181961 & -5.186782 & 0.010674 \\
\hline C & 7.006946 & -3.856735 & 0.400235 \\
\hline C & 3.933728 & 2.869672 & -0.593319 \\
\hline C & 4.103389 & 3.217536 & -1.944067 \\
\hline C & 3.902431 & 4.533652 & -2.371000 \\
\hline C & 3.532602 & 5.520334 & -1.451727 \\
\hline C & 3.357351 & 5.181228 & -0.105305 \\
\hline $\mathrm{C}$ & 3.545950 & 3.863586 & ๑. 319048 \\
\hline C & 3.933851 & -2.870837 & 0.586947 \\
\hline C & 4.105884 & -3.218702 & 1.937400 \\
\hline C & 3.905330 & -4.534745 & 2.364752 \\
\hline C & 3.533526 & -5.521355 & 1. 446199 \\
\hline C & 3.355910 & -5.182249 & 0.100086 \\
\hline C & 3.544094 & -3.864678 & -0.324660 \\
\hline C & -3.933728 & -2.869672 & ๑. 593319 \\
\hline C & -4.103389 & -3.217536 & 1.944067 \\
\hline C & -3.902431 & -4.533652 & 2.371000 \\
\hline C & -3.532602 & -5.520334 & 1.451727 \\
\hline C & -3.357351 & -5.181228 & 0.105305 \\
\hline C & -3.545950 & -3.863586 & -0.319048 \\
\hline C & -3.933851 & 2.870837 & -0.586947 \\
\hline C & -4.105884 & 3.218702 & -1.937400 \\
\hline C & -3.905330 & 4.534745 & -2.364752 \\
\hline C & -3.533526 & 5.521355 & -1.446199 \\
\hline C & -3.355910 & 5.182249 & -0.100086 \\
\hline C & -3.544094 & 3.864678 & $\odot .324660$ \\
\hline C & -6.628112 & 2.882698 & 0.537280 \\
\hline C & -7.006946 & 3.856735 & -0.400235 \\
\hline C & -7.181961 & 5.186782 & -0.010674 \\
\hline C & $-7.0 \odot 1972$ & 5.559177 & 1. 326511 \\
\hline C & -6.640098 & 4.593518 & 2.270850 \\
\hline C & -6.452447 & 3.264708 & 1.877923 \\
\hline $\mathrm{H}$ & -11.709590 & -3.276803 & -0.906870 \\
\hline $\mathrm{H}$ & -9.243601 & -3.232409 & -0.866090 \\
\hline $\mathrm{H}$ & -13.065894 & -1.285398 & $-\odot .351207$ \\
\hline $\mathrm{H}$ & -13.064884 & 1.289851 & 0.370913 \\
\hline $\mathrm{H}$ & -9.241116 & 3.235408 & ๑. 880344 \\
\hline $\mathrm{H}$ & -1.217274 & -3.239985 & 0.882820 \\
\hline $\mathrm{H}$ & 1.217742 & -3.240390 & 0.880669 \\
\hline $\mathrm{H}$ & -1.217742 & 3.240390 & $-\odot .880669$ \\
\hline $\mathrm{H}$ & 1.217274 & 3.239985 & -0.882820 \\
\hline $\mathrm{H}$ & -11.707038 & 3.280747 & 0.924625 \\
\hline
\end{tabular}




\begin{tabular}{|c|c|c|c|}
\hline$H$ & 9.241116 & -3.235408 & -0.880344 \\
\hline $\mathrm{H}$ & 11.707038 & -3.280747 & -0.924625 \\
\hline $\mathrm{H}$ & 13.064884 & -1.289851 & -0.370913 \\
\hline $\mathrm{H}$ & 9.243601 & 3.232409 & 0.866090 \\
\hline $\mathrm{H}$ & 11.709590 & 3.276803 & 0.906870 \\
\hline $\mathrm{H}$ & 13.065894 & 1.285398 & 0. 351207 \\
\hline $\mathrm{H}$ & -7.161369 & -3.567147 & 1.455168 \\
\hline $\mathrm{H}$ & -7.467087 & -5.933791 & ๑. 767912 \\
\hline $\mathrm{H}$ & -7.152365 & -6.597832 & -1.619727 \\
\hline $\mathrm{H}$ & -6.513831 & -4.872034 & -3.309607 \\
\hline $\mathrm{H}$ & -6.183724 & -2.508746 & -2.611793 \\
\hline $\mathrm{H}$ & -4.386758 & -2.447425 & 2.667813 \\
\hline $\mathrm{H}$ & -3.398364 & -3.603069 & -1.370343 \\
\hline $\mathrm{H}$ & -3.069137 & -5.947547 & -0.620623 \\
\hline $\mathrm{H}$ & -3.379716 & -6.551811 & 1.783569 \\
\hline $\mathrm{H}$ & -4.034024 & -4.787699 & 3.427356 \\
\hline $\mathrm{H}$ & -7.162746 & 3.569525 & -1.443414 \\
\hline $\mathrm{H}$ & -7.466523 & 5.936199 & -0.755407 \\
\hline $\mathrm{H}$ & -7.147556 & 6.599887 & 1.631776 \\
\hline $\mathrm{H}$ & -6.506734 & 4.873698 & 3.320389 \\
\hline $\mathrm{H}$ & -6.178562 & 2.510384 & 2.621773 \\
\hline $\mathrm{H}$ & -4.390952 & 2.448688 & -2.660577 \\
\hline $\mathrm{H}$ & -4.038927 & 4.788829 & -3.420845 \\
\hline $\mathrm{H}$ & -3.381008 & 6.552792 & -1.778330 \\
\hline $\mathrm{H}$ & -3.066123 & 5.948515 & 0.625289 \\
\hline $\mathrm{H}$ & -3.394603 & 3.604152 & 1.375689 \\
\hline $\mathrm{H}$ & 4.386758 & 2.447425 & -2.667813 \\
\hline $\mathrm{H}$ & 4.034024 & 4.787699 & -3.427356 \\
\hline $\mathrm{H}$ & 3.379716 & 6.551811 & -1.783569 \\
\hline $\mathrm{H}$ & 3.069137 & 5.947547 & 0.620623 \\
\hline $\mathrm{H}$ & 3.398364 & 3.603069 & 1.370343 \\
\hline $\mathrm{H}$ & 7.161369 & 3.567147 & -1.455168 \\
\hline $\mathrm{H}$ & 7.467087 & 5.933791 & -0.767912 \\
\hline $\mathrm{H}$ & 7.152365 & 6.597832 & 1.619727 \\
\hline $\mathrm{H}$ & 6.513831 & 4.872034 & 3.309607 \\
\hline $\mathrm{H}$ & 6.183724 & 2.508746 & 2.611793 \\
\hline $\mathrm{H}$ & 4.390952 & -2.448688 & 2.660577 \\
\hline $\mathrm{H}$ & 4.038927 & -4.788829 & 3.420845 \\
\hline $\mathrm{H}$ & 3.381008 & -6.552792 & 1.778330 \\
\hline $\mathrm{H}$ & 3.066123 & -5.948515 & -0.625289 \\
\hline $\mathrm{H}$ & 3.394603 & -3.604152 & -1.375689 \\
\hline $\mathrm{H}$ & 7.162746 & -3.569525 & 1.443414 \\
\hline $\mathrm{H}$ & 7.466523 & -5.936199 & $\odot .755407$ \\
\hline $\mathrm{H}$ & 7.147556 & -6.599887 & -1.631776 \\
\hline $\mathrm{H}$ & 6.506734 & -4.873698 & -3.320389 \\
\hline $\mathrm{H}$ & 6.178562 & -2.510384 & -2.621773 \\
\hline \multicolumn{4}{|c|}{$E=-3765.208906$} \\
\hline
\end{tabular}


B conformation, electronic ground state, anionic form, B3LYP/SV (P)

\section{( $D_{2}$ symmetry)}

$\begin{array}{lrrr}\text { C } & -6.475484 & -3.263873 & -1.860103 \\ \text { C } & -6.640846 & -2.878789 & -0.518890 \\ \text { C } & -7.008574 & -3.859510 & 0.415961 \\ \text { C } & -7.186416 & -5.189421 & 0.026874 \\ \text { C } & -7.016229 & -5.561257 & -1.311400 \\ \text { C } & -6.661982 & -4.592068 & -2.255189 \\ \text { C } & -6.547457 & -1.424724 & -0.161173 \\ \text { C } & -5.290762 & -0.731431 & 0.019472 \\ \text { C } & -5.290552 & 0.733105 & -0.010374 \\ \text { C } & -6.546687 & 1.426849 & 0.172414 \\ \text { C } & -7.735391 & 0.712993 & 0.145522 \\ \text { C } & -7.735853 & -0.710416 & -0.132454 \\ \text { C } & -4.041903 & 1.423647 & -0.204004 \\ \text { C } & -2.843506 & 0.717702 & -0.156396 \\ \text { C } & -2.843473 & -0.716672 & 0.161535 \\ \text { C } & -4.041979 & -1.422317 & 0.210974 \\ \text { C } & -1.448962 & -1.151916 & 0.309586 \\ \text { C } & -0.689498 & 0.000131 & 0.000639 \\ \text { C } & -1.449102 & 1.152452 & -0.306942 \\ \text { C } & -0.708106 & -2.309968 & 0.648923 \\ \text { C } & 0.708435 & -2.310225 & 0.647645 \\ \text { C } & 1.449102 & -1.152452 & 0.306942 \\ \text { C } & 0.689498 & -0.000131 & -0.000639 \\ \text { C } & 1.448962 & 1.151916 & -0.309586 \\ \text { C } & 2.843473 & 0.716672 & -0.161535 \\ \text { C } & 2.843506 & -0.717702 & 0.156396 \\ \text { C } & 4.041903 & -1.423647 & 0.204004 \\ \text { C } & 5.290552 & -0.733105 & 0.010374 \\ \text { C } & 5.290762 & 0.731431 & -0.019472 \\ \text { C } & 4.041979 & 1.422317 & -0.210974 \\ \text { C } & 6.546687 & -1.426849 & -0.172414 \\ \text { C } & 7.735391 & -0.712993 & -0.145522 \\ \text { C } & 7.735853 & 0.710416 & 0.132454 \\ \text { C } & 6.547457 & 1.424724 & 0.161173\end{array}$




\begin{tabular}{|c|c|c|c|}
\hline $\mathrm{C}$ & 9.141397 & 1.141811 & ๑. 281430 \\
\hline C & 9.939702 & -0.001634 & -0.008449 \\
\hline C & 9.140537 & -1.144824 & -0.296964 \\
\hline C & 11.351946 & $-\odot .0 \odot 1847$ & $-\odot .0 \odot 9717$ \\
\hline C & 11.987669 & -1.229982 & -0.352692 \\
\hline C & 11.218166 & -2.343407 & -0.661522 \\
\hline $\mathrm{C}$ & 9.798163 & -2.324964 & -0.637292 \\
\hline $\mathrm{C}$ & 11.988652 & 1. 226106 & 0.332087 \\
\hline C & 11.220037 & 2.339779 & $\odot .642227$ \\
\hline $\mathrm{C}$ & 9.799987 & 2.321769 & ๑. 620520 \\
\hline C & -0.708435 & 2.310225 & -0.647645 \\
\hline C & 0.708106 & 2.309968 & -0.648923 \\
\hline $\mathrm{C}$ & -9.140537 & 1.144824 & ๑. 296964 \\
\hline $\mathrm{C}$ & -9.939702 & ๑ . 001634 & ๑ . 008449 \\
\hline C & -9.141397 & -1.141811 & -0.281430 \\
\hline$C$ & -11.351946 & ๑ . 001847 & $\odot .009717$ \\
\hline C & -11.988652 & -1.226106 & -0.332087 \\
\hline C & -11.220037 & -2.339779 & -0.642227 \\
\hline $\mathrm{C}$ & -9.799987 & -2.321769 & -0.620520 \\
\hline C & -11.987669 & 1.229982 & ๑. 352692 \\
\hline C & -11.218166 & 2.343407 & $\odot .661522$ \\
\hline C & -9.798163 & 2.324964 & $\odot .637292$ \\
\hline $\mathrm{C}$ & 6.640846 & 2.878789 & ๑. 518890 \\
\hline C & 6.475484 & 3.263873 & 1.860103 \\
\hline C & 6.661982 & 4.592068 & 2.255189 \\
\hline C & 7.016229 & 5.561257 & 1.311400 \\
\hline C & 7.186416 & 5.189421 & -0.026874 \\
\hline $\mathrm{C}$ & 7.008574 & 3.859510 & -0.415961 \\
\hline C & 6.638845 & -2.880861 & -0.530656 \\
\hline C & 6.470581 & -3.265588 & -1.871613 \\
\hline C & 6.655847 & -4.593754 & -2.267382 \\
\hline C & 7.011726 & -5.563274 & -1.324551 \\
\hline C & 7.184769 & -5.191803 & 0.013461 \\
\hline C & 7.008165 & -3.861924 & 0.403209 \\
\hline C & 3.940940 & 2.868222 & -0.598600 \\
\hline C & 4.115695 & 3.230912 & -1.945028 \\
\hline C & 3.922883 & 4.550115 & -2.366314 \\
\hline C & 3.551044 & 5.533276 & -1.444075 \\
\hline C & 3.366490 & 5.183421 & -0.101868 \\
\hline $\mathrm{C}$ & 3.550403 & 3.862142 & 0.312879 \\
\hline C & 3.941278 & -2.869404 & ๑. 592264 \\
\hline $\mathrm{C}$ & 4.118950 & -3.231789 & 1.938395 \\
\hline $\mathrm{C}$ & 3.926759 & -4.550839 & 2.360451 \\
\hline C & 3.552678 & -5.534163 & 1.439296 \\
\hline C & 3.365268 & -5.184621 & $\odot .097400$ \\
\hline C & 3.548541 & -3.863491 & -0.318093 \\
\hline C & -3.940940 & -2.868222 & 0.598600 \\
\hline
\end{tabular}




\begin{tabular}{|c|c|c|c|}
\hline C & -4.115695 & -3.230912 & 1.945028 \\
\hline C & -3.922883 & -4.550115 & 2.366314 \\
\hline C & -3.551044 & -5.533276 & 1.444075 \\
\hline C & -3.366490 & -5.183421 & ๑. 101868 \\
\hline C & -3.550403 & -3.862142 & -0.312879 \\
\hline C & -3.941278 & 2.869404 & -0.592264 \\
\hline C & -4.118950 & 3.231789 & -1.938395 \\
\hline C & -3.926759 & 4.550839 & -2.360451 \\
\hline C & -3.552678 & 5.534163 & -1.439296 \\
\hline C & -3.365268 & 5.184621 & -0.097400 \\
\hline C & -3.548541 & 3.863491 & ๑. 318093 \\
\hline C & -6.638845 & 2.880861 & ๑. 530656 \\
\hline C & -7.008165 & 3.861924 & -0.403209 \\
\hline C & -7.184769 & 5.191803 & -0.013461 \\
\hline C & -7.011726 & 5.563274 & 1.324551 \\
\hline C & -6.655847 & 4.593754 & 2.267382 \\
\hline C & -6.470581 & 3.265588 & 1.871613 \\
\hline $\mathrm{H}$ & -11.721899 & -3.275722 & -0.911503 \\
\hline $\mathrm{H}$ & -9.257098 & -3.232639 & -0.872279 \\
\hline $\mathrm{H}$ & -13.082284 & -1.284356 & -0.352345 \\
\hline $\mathrm{H}$ & -13.081246 & 1.288566 & 0.374882 \\
\hline $\mathrm{H}$ & -9.254540 & 3.235646 & ๑. 888155 \\
\hline $\mathrm{H}$ & -1.212118 & -3.237266 & ๑. 926512 \\
\hline $\mathrm{H}$ & 1.212608 & -3.237701 & ๑. 924336 \\
\hline $\mathrm{H}$ & -1.212608 & 3.237701 & -0.924336 \\
\hline $\mathrm{H}$ & 1.212118 & 3.237266 & -0.926512 \\
\hline $\mathrm{H}$ & -11.719265 & 3.279491 & 0.931727 \\
\hline $\mathrm{H}$ & 9.254540 & -3.235646 & -0.888155 \\
\hline $\mathrm{H}$ & 11.719265 & -3.279491 & -0.931727 \\
\hline $\mathrm{H}$ & 13.081246 & -1.288566 & -0.374882 \\
\hline $\mathrm{H}$ & 9.257098 & 3.232639 & ๑. 872279 \\
\hline $\mathrm{H}$ & 11.721899 & 3.275722 & $\odot .911503$ \\
\hline $\mathrm{H}$ & 13.082284 & 1.284356 & 0.352345 \\
\hline $\mathrm{H}$ & -7.153749 & -3.572347 & 1.460503 \\
\hline $\mathrm{H}$ & -7.461219 & -5.940562 & $\odot .774467$ \\
\hline $\mathrm{H}$ & -7.158608 & -6.603184 & -1.616826 \\
\hline $\mathrm{H}$ & -6.530581 & -4.870346 & -3.306269 \\
\hline $\mathrm{H}$ & -6.203780 & -2.506098 & -2.601056 \\
\hline $\mathrm{H}$ & -4.396940 & -2.461093 & 2.669977 \\
\hline $\mathrm{H}$ & -3.388510 & - 3.590859 & -1.359303 \\
\hline $\mathrm{H}$ & -3.069931 & -5.944141 & -0.627563 \\
\hline $\mathrm{H}$ & -3.399416 & -6.567545 & 1.770472 \\
\hline $\mathrm{H}$ & -4.058569 & -4.809586 & 3.421740 \\
\hline $\mathrm{H}$ & -7.155571 & 3.575048 & -1.447517 \\
\hline $\mathrm{H}$ & -7.460851 & 5.943207 & -0.760317 \\
\hline $\mathrm{H}$ & - 7.153148 & 6.605175 & 1.630514 \\
\hline $\mathrm{H}$ & -6.522202 & 4.871745 & 3.318255 \\
\hline
\end{tabular}




$\begin{array}{rrrr}\mathrm{H} & -6.197597 & 2.507560 & 2.611837 \\ \mathrm{H} & -4.402011 & 2.461857 & -2.662518 \\ \mathrm{H} & -4.064739 & 4.810066 & -3.415641 \\ \mathrm{H} & -3.401555 & 6.568317 & -1.766293 \\ \mathrm{H} & -3.066961 & 5.945470 & 0.631183 \\ \mathrm{H} & -3.384404 & 3.592455 & 1.364231 \\ \mathrm{H} & 4.396940 & 2.461093 & -2.669977 \\ \mathrm{H} & 4.058569 & 4.809586 & -3.421740 \\ \mathrm{H} & 3.399416 & 6.567545 & -1.770472 \\ \mathrm{H} & 3.069931 & 5.944141 & 0.627563 \\ \mathrm{H} & 3.388510 & 3.590859 & 1.359303 \\ \mathrm{H} & 7.153749 & 3.572347 & -1.460503 \\ \mathrm{H} & 7.461219 & 5.940562 & -0.774467 \\ \mathrm{H} & 7.158608 & 6.603184 & 1.616826 \\ \mathrm{H} & 6.530581 & 4.870346 & 3.306269 \\ \mathrm{H} & 6.203780 & 2.506098 & 2.601056 \\ \mathrm{H} & 4.402011 & -2.461857 & 2.662518 \\ \mathrm{H} & 4.064739 & -4.810066 & 3.415641 \\ \mathrm{H} & 3.401555 & -6.568317 & 1.766293 \\ \mathrm{H} & 3.066961 & -5.945470 & -0.631183 \\ \mathrm{H} & 3.384404 & -3.592455 & -1.364231 \\ \mathrm{H} & 7.155571 & -3.575048 & 1.447517 \\ \mathrm{H} & 7.460851 & -5.943207 & 0.760317 \\ \mathrm{H} & 7.153148 & -6.605175 & -1.630514 \\ \mathrm{H} & 6.522202 & -4.871745 & -3.318255 \\ \mathrm{H} & 6.197597 & -2.507560 & -2.611837 \\ & \mathrm{E}=-3765.484283 & \\ & & & \end{array}$

Data for Figure 5. IR spectra calculated for ONIOMB3LYP/SV (P).

A conformation, electronic ground state, anionic form, B3LYP/SV ( $P)$, ( $D_{2}$ symmetry)

$$
\begin{array}{lr}
\text { Freqency } & \text { Intenisty } \\
\left(\mathrm{cm}^{-1}\right) & \left(\mathrm{km} \cdot \mathrm{mol}^{-1}\right)
\end{array}
$$




\begin{tabular}{|c|c|}
\hline 17.7203 & 0.0030 \\
\hline 23.8748 & 0.0000 \\
\hline 25.6822 & 0.0005 \\
\hline 30.1811 & 0.0000 \\
\hline 32.3521 & 0.0086 \\
\hline 33.2629 & 0.0000 \\
\hline 34.1011 & 0.0043 \\
\hline 34.2193 & 0.0147 \\
\hline 41.3809 & 0.1568 \\
\hline 47.8056 & 0.0083 \\
\hline 47.9186 & 0.0000 \\
\hline 48.5032 & 0.0953 \\
\hline 48.6961 & 0.0098 \\
\hline 52.1442 & 0.0000 \\
\hline 52.4790 & 0.0090 \\
\hline 52.6672 & 0.0078 \\
\hline 52.8165 & 0.0040 \\
\hline 53.6303 & 0.0000 \\
\hline 57.2279 & 0.0423 \\
\hline 58.7156 & 0.0555 \\
\hline 59.4139 & 0.0000 \\
\hline 62.7489 & 0.0359 \\
\hline 68.3766 & $\odot .2281$ \\
\hline 70.6111 & 0.1620 \\
\hline 71.5883 & $\odot .0849$ \\
\hline 71.7222 & 0.2178 \\
\hline 78.7381 & ๑. . 0917 \\
\hline 79.5244 & $0.000 \odot$ \\
\hline 79.6655 & 0.0001 \\
\hline 79.9400 & $\odot .0346$ \\
\hline 86.3668 & 0.1275 \\
\hline 103.0114 & 0.0654 \\
\hline 120.8722 & $\odot .000 \odot$ \\
\hline 128.4615 & 0.0720 \\
\hline 135.7256 & 0.0510 \\
\hline 138.9841 & 0.0000 \\
\hline 142.8483 & ๑. 2998 \\
\hline 153.1917 & 1.3047 \\
\hline 164.3126 & $\odot .4704$ \\
\hline 171.1053 & 0.0855 \\
\hline 172.7339 & 4.9895 \\
\hline 179.4551 & ๑. . 0000 \\
\hline 185.8368 & 0.0173 \\
\hline 187.4978 & 1.5483 \\
\hline 204.9550 & 0.0412 \\
\hline 205.3496 & 3.2667 \\
\hline
\end{tabular}




207.5757
209.5574
214.0113
216.7612
217.0212
217.7683
218.9935
220.6534
227.1823
232.0556
232.7015
234.0067
246.0271
248.1036
261.5654
273.8114
292.2527
292.3559
320.8088
325.7136
328.2474
339.2742
346.1614
365.1780
365.2602
365.2613
365.3625
367.9623
367.9682
367.9953
368.0328
383.7531
404.8828
407.4949
426.3886
426.8718
433.8066
433.8404
445.5575
460.4965
465.6489
474.1085
474.5285
476.5178
485.39574
488.3431

$\odot .1960$

0.0000

0.0431

0.1089

๑. 1547

0.1384

0.0000

0.0326

0.0119

0.0000

$\odot .0059$

0.0000

0.1336

0.0000

$\odot .0235$

1. 6213

0.1439

0.1465

0.0017

0.0000

0.0034

0.1842

2.0353

0.0253

0.3011

0.0000

0.0430

0.0011

0.0000

0.0025

0.0048

0.3781

2.7365

0.0000

$\odot .2305$

0.0000

0.2793

0.7245

8.0310

$\odot .0209$

0.0325

0.6491

$\odot .6116$ 0.0943

1. 2822

0.0000

0.2472 


\begin{tabular}{|c|c|}
\hline 491.8764 & 4.4856 \\
\hline 507.6394 & $\odot .0000$ \\
\hline 508.3928 & 1.3013 \\
\hline 517.0456 & 0.0000 \\
\hline 519.3497 & 20.3919 \\
\hline 523.9385 & 2.3013 \\
\hline 529.5776 & $\odot .2241$ \\
\hline 535.9403 & 0.0000 \\
\hline 541.4228 & 0.1829 \\
\hline 545.0607 & 0.8094 \\
\hline 547.1636 & $\odot .0000$ \\
\hline 550.1977 & 0.3272 \\
\hline 554.5188 & 0.1790 \\
\hline 556.0614 & 15.2824 \\
\hline 586.7887 & 0.0000 \\
\hline 594.8216 & 5.6190 \\
\hline 600.6733 & 0.0000 \\
\hline 605.5212 & 0.3774 \\
\hline 615.7394 & 0.0125 \\
\hline 619.4478 & 5.6836 \\
\hline 619.6887 & 0.0000 \\
\hline 622.0231 & 3.5753 \\
\hline 623.8493 & 0.5595 \\
\hline 625.2324 & 0.1635 \\
\hline 630.7691 & 0.0038 \\
\hline 631.4363 & 3.8894 \\
\hline 634.5154 & 23.8472 \\
\hline 636.2114 & $\odot .000 \odot$ \\
\hline 643.5025 & 0.0000 \\
\hline 646.6189 & 4.0063 \\
\hline 650.9434 & 0.4430 \\
\hline 651.5349 & $\odot .000 \odot$ \\
\hline 651.6971 & 0.0017 \\
\hline 653.3712 & 0.0145 \\
\hline 653.4357 & 0.0055 \\
\hline 654.3277 & $\odot .0 \odot \odot \odot$ \\
\hline 654.4378 & 6.2974 \\
\hline 657.7674 & 5.4540 \\
\hline 661.0266 & 243.6441 \\
\hline 661.4053 & 0.0000 \\
\hline 667.6074 & $\odot .000 \odot$ \\
\hline 676.0726 & 0.2330 \\
\hline 676.6607 & 0.0000 \\
\hline 681.6385 & 1.1591 \\
\hline 682.9938 & 0.7997 \\
\hline 686.2066 & 1.5859 \\
\hline 687.6113 & 0.0000 \\
\hline
\end{tabular}




\begin{tabular}{|c|c|}
\hline 691.6569 & 2.6267 \\
\hline 701.5789 & $\odot .9646$ \\
\hline 710.0269 & 144.5664 \\
\hline 731.3117 & 36.9138 \\
\hline 735.6441 & 6.2911 \\
\hline 739.9524 & 3.9429 \\
\hline 745.0997 & 0.0000 \\
\hline 747.1227 & 6.1972 \\
\hline 751.1039 & 18.9534 \\
\hline 752.2529 & 0.4664 \\
\hline 756.2921 & 0.1148 \\
\hline 763.4525 & 0.1721 \\
\hline 769.6008 & 0.0000 \\
\hline 777.0311 & 0.6812 \\
\hline 780.9084 & 24.0555 \\
\hline 780.9090 & 89.2810 \\
\hline 784.0473 & 4.2241 \\
\hline 794.6213 & 0.0000 \\
\hline $795.0 \odot 59$ & 189.9279 \\
\hline 796.3949 & 0.0000 \\
\hline 804.0372 & 0.7429 \\
\hline 815.6966 & 4.0308 \\
\hline 816.5595 & 0.0782 \\
\hline 817.5796 & 1.4908 \\
\hline 821.1634 & 1.9406 \\
\hline 821.2198 & ๑. . $00 \odot \odot$ \\
\hline 826.7656 & 0.3038 \\
\hline 839.7464 & $\odot .00 \odot \odot$ \\
\hline 840.4047 & 0.0392 \\
\hline 851.1673 & 2.0821 \\
\hline 853.5552 & 26.4906 \\
\hline 855.3313 & 6.8449 \\
\hline 857.7196 & 7.3328 \\
\hline 859.0392 & 23.5786 \\
\hline 861.1082 & 0.0000 \\
\hline $861 \cdot 1243$ & 7.2828 \\
\hline 861.4496 & ๑. . 0628 \\
\hline 861.5818 & 2.5200 \\
\hline 863.1237 & 0.0503 \\
\hline 863.5791 & 1.0059 \\
\hline 864.2598 & $\odot . \odot \odot \odot \odot$ \\
\hline 864.3670 & 0.8155 \\
\hline 865.3071 & 10.0474 \\
\hline 865.6046 & 0.7379 \\
\hline 872.8690 & 0.1070 \\
\hline 875.2440 & $\odot .0 \odot ० \odot$ \\
\hline 883.2836 & 0.0000 \\
\hline
\end{tabular}




\begin{tabular}{|c|c|}
\hline 895.5309 & 3.9414 \\
\hline 912.8591 & 0.0447 \\
\hline 918.0317 & 0.0005 \\
\hline 918.0327 & 0.0000 \\
\hline 920.1512 & 8.9030 \\
\hline 929.6399 & 0.1807 \\
\hline 933.5820 & 0.0000 \\
\hline 934.3543 & 3.7750 \\
\hline 934.6730 & 1.3902 \\
\hline 934.6805 & $\odot .0000$ \\
\hline 934.7116 & 6.3838 \\
\hline 940.3154 & 0.5766 \\
\hline 941.4858 & 0.0069 \\
\hline 943.1154 & ๑. 9983 \\
\hline 944.4595 & 8.8743 \\
\hline 947.8026 & 0.0000 \\
\hline 955.7663 & 0.1754 \\
\hline 968.4960 & 0.0000 \\
\hline 968.5460 & ๑. 8229 \\
\hline 968.5528 & 0.0325 \\
\hline 968.6283 & 0.0063 \\
\hline 969.7036 & 0.0000 \\
\hline 969.7064 & $\odot .0032$ \\
\hline 969.7344 & 0.0048 \\
\hline 969.7430 & 0.2061 \\
\hline 972.2512 & 1.9738 \\
\hline 973.0546 & $\odot .0 \odot \odot \odot$ \\
\hline 973.0561 & 0.0073 \\
\hline 975.4845 & 0.0000 \\
\hline 976.7492 & 0.0048 \\
\hline 982.2014 & 0.2091 \\
\hline 983.1987 & 2.4460 \\
\hline 983.2119 & 0.7245 \\
\hline 989.0922 & 0.0000 \\
\hline 989.7381 & 0.0124 \\
\hline 991.2891 & $\odot .0000$ \\
\hline 991.4282 & 0.0114 \\
\hline 991.5935 & 0.0273 \\
\hline 991.6993 & 0.0384 \\
\hline 992.1698 & 0.0051 \\
\hline 992.5862 & $\odot .0 \odot \odot \odot$ \\
\hline 993.1635 & 0.1699 \\
\hline 997.3023 & 1.8812 \\
\hline 1015.8603 & 0.8079 \\
\hline 1030.9750 & 1.4114 \\
\hline 1033.2339 & $\odot .0000$ \\
\hline 1062.9894 & 0.5906 \\
\hline
\end{tabular}




\begin{tabular}{|c|c|}
\hline 1063.7885 & 14.1490 \\
\hline 1066.0658 & 1.7767 \\
\hline 1078.9831 & 0.0000 \\
\hline 1084.7946 & $\odot .0019$ \\
\hline 1091.7462 & 0.9882 \\
\hline 1107.2880 & 0.0000 \\
\hline 1107.8683 & 0.1823 \\
\hline 1111.4525 & 0.0000 \\
\hline 1114.2910 & 0.0335 \\
\hline 1130.6981 & 0.0000 \\
\hline 1135.0891 & 1.2588 \\
\hline 1139.4081 & ๑. 2861 \\
\hline 1143.1456 & 1.2969 \\
\hline 1143.1585 & 0.0007 \\
\hline 1144.0220 & 0.5962 \\
\hline 1144.4302 & 0.0000 \\
\hline 1144.5446 & 0.4174 \\
\hline 1144.8081 & 0.0000 \\
\hline 1145.0049 & 0.2494 \\
\hline 1146.2131 & 3.8774 \\
\hline 1148.5663 & 0.0392 \\
\hline 1149.6107 & 0.0802 \\
\hline 1151.5514 & 0.8439 \\
\hline 1151.5764 & ๑. 9152 \\
\hline 1153.8895 & 0.0951 \\
\hline 1153.9182 & 0.0000 \\
\hline 153.9685 & 3.0289 \\
\hline 1166.4329 & 0.1454 \\
\hline 1170.2409 & 0.0000 \\
\hline 1171.4886 & 0.2920 \\
\hline 1178.6903 & 0.0852 \\
\hline 1178.7343 & 0.0000 \\
\hline 181.4808 & 0.5837 \\
\hline 1181.4813 & 0.0534 \\
\hline 1181.5076 & 0.0151 \\
\hline 181.5445 & 0.0000 \\
\hline 1181.8265 & 0.0217 \\
\hline 1181.8619 & 0.0334 \\
\hline 1181.8777 & 1.0337 \\
\hline 1181.8783 & 0.0000 \\
\hline 1186.4561 & 4.0155 \\
\hline 1192.5644 & 0.4580 \\
\hline 1192.9328 & 0.0012 \\
\hline 1201.2695 & 0.0000 \\
\hline 1210.8756 & 0.0273 \\
\hline 1210.9591 & 0.9226 \\
\hline 1212.2919 & 0.1483 \\
\hline
\end{tabular}




1212.3291
1213.6478
1214.1887
1214.2349
1214.6952
1218.7260
1220.4268
1221.5244
1222.5184
1238.3495
1247.6189
1248.9798
1252.7269
1260.7468
1262.3270
1272.4416
1275.8837
1277.2260
1297.0923
1297.6048
1297.6254
1297.6282
1297.8283
1297.8395
1297.9142
1297.9212
1298.5345
1301.7375
1301.7433
1305.6660
1338.7801
1342.6498
1342.6586
1342.8710
1343.8217
1344.0261
1344.0514
1344.1292
1344.7120
1358.4921
1377.7080
1383.3976
1383.4421
1387.4579
1394.6205
1398.8994
1401.4220

6.9606

๑. 2462

1. 2454

0.0000

0.0000

$\odot .0266$

0.0000

0.2161

0.0000

10.8150

0.0000

4.2779

0.2856

3.2135

0.1002

0.9212

0.0030

37.9646

0.0000

๑. 3822

0.3396

0.0155

0.0000

0.0188

0.0038

$\odot .000 \odot$

0.0000

24.3910

7. 3601

19.2855

0.0000

1. 0133

0.0217

2.7883

0.0000

0.0012

0.0028

1. 6879

0.0000

248.3390

0.8583

0.4658

0.0000

4.2579

0.0000

246.1751

0.0000 


\begin{tabular}{|c|c|}
\hline 1403.8590 & 0.2318 \\
\hline 1429.1007 & 0.0000 \\
\hline 1430.2342 & 0.2773 \\
\hline 1449.8572 & 13.1813 \\
\hline 1460.9562 & 3.5163 \\
\hline 1462.5010 & 104.4682 \\
\hline 1469.2672 & 0.0000 \\
\hline 1469.4273 & ○. 2987 \\
\hline 1477.7200 & 5.8438 \\
\hline 1478.0352 & 0.6383 \\
\hline 1483.1401 & 1.0365 \\
\hline 1483.3750 & 48.4813 \\
\hline 1485.3920 & 0.0000 \\
\hline 1504.3836 & 1.0772 \\
\hline 1517.3479 & 1.5411 \\
\hline 1521.4397 & 0.0777 \\
\hline 1521.5029 & 0.0000 \\
\hline 1546.4115 & 2.8716 \\
\hline 1546.4252 & 2.2146 \\
\hline 1546.8575 & 3.4968 \\
\hline 1546.8608 & 0.0000 \\
\hline 1546.8764 & 0.1631 \\
\hline 546.8896 & 57.1898 \\
\hline 1547.0340 & 0.8078 \\
\hline 1547.0508 & 0.0000 \\
\hline 1552.5957 & 0.1759 \\
\hline 1555.9074 & 0.0000 \\
\hline 1592.3001 & 10.2511 \\
\hline 1592.8239 & 0.0000 \\
\hline 1605.9235 & 5.1660 \\
\hline 1607.1915 & 3.3693 \\
\hline 1608.2223 & 8.6528 \\
\hline 1608.4271 & 0.2509 \\
\hline 1616.2787 & 57.2910 \\
\hline 1616.3175 & 0.0000 \\
\hline 1620.0347 & 2.6512 \\
\hline 1621.5189 & 2.0731 \\
\hline 1621.6140 & 1.3134 \\
\hline 1629.7816 & 22.3685 \\
\hline 1630.1135 & 0.0000 \\
\hline 1635.7421 & 0.0369 \\
\hline 1639.8490 & 0.1545 \\
\hline 1663.6541 & $\odot .6593$ \\
\hline 665.4202 & 5.4384 \\
\hline 1666.3777 & $\odot .9514$ \\
\hline 1669.6856 & 0.0000 \\
\hline 1670.1990 & 51.3900 \\
\hline
\end{tabular}




\begin{tabular}{|c|c|}
\hline 1704.5719 & 2.8879 \\
\hline 1704.6742 & 0.7047 \\
\hline 1738.7153 & 0.0135 \\
\hline 1738.7193 & 0.0026 \\
\hline 1739.0348 & 0.0000 \\
\hline 1739.0585 & $\odot .0 \odot \odot \odot$ \\
\hline 1739.0618 & 0.0042 \\
\hline 1739.0626 & 0.0014 \\
\hline 1739.2422 & 0.0000 \\
\hline 1739.2570 & 0.1052 \\
\hline 1743.8092 & 0.0000 \\
\hline 1759.2497 & 0.0042 \\
\hline 1759.2500 & 0.2643 \\
\hline 1759.3805 & 0.7241 \\
\hline 1759.3822 & $\odot .000 \odot$ \\
\hline 1759.5850 & 0.0024 \\
\hline 1759.5947 & 0.4179 \\
\hline 1759.6958 & 0.3674 \\
\hline 1759.7528 & 0.0000 \\
\hline 1770.4594 & 0.0000 \\
\hline 1770.4595 & 11.7733 \\
\hline 1788.6130 & 3.0922 \\
\hline 1788.6135 & 10.2320 \\
\hline 1817.6552 & 57.6375 \\
\hline 1817.7395 & $\odot .0000$ \\
\hline 3195.9442 & 7.6726 \\
\hline 3196.0875 & 0.0652 \\
\hline 3211.3084 & 40.6632 \\
\hline 3211.7108 & 0.0000 \\
\hline 3218.4007 & 61.6341 \\
\hline 3218.4060 & $\odot .0000$ \\
\hline 3218.4271 & 6.8484 \\
\hline 3218.4285 & 24.8191 \\
\hline 3218.8737 & 2.3822 \\
\hline 3218.8756 & 0.0279 \\
\hline 3218.8927 & 0.4351 \\
\hline 3218 . 8952 & 0.0000 \\
\hline 3219.2595 & 1.2563 \\
\hline 3219.2599 & 0.0556 \\
\hline 3219.2653 & 0.0149 \\
\hline 3219.2665 & 0.0000 \\
\hline 3222.6530 & 12.9607 \\
\hline 3222.6545 & 0.0000 \\
\hline 3222.6952 & 16.0876 \\
\hline 3222.7015 & $\odot .2453$ \\
\hline 3222.9628 & 0.0000 \\
\hline 3222.9678 & $\odot .1355$ \\
\hline
\end{tabular}




3222.9942
3223.0019
3227.5391
3227.5468
3227.5837
3227.5868
3228.0064
3228.1010
3228.1165
3228.1687
3228.1693
3228.2165
3228.5496
3228.5917
3232.6966
3232.7052
3232.7268
3232.7303
3232.9620
3233.0046
3233.1270
3233.2485
3240.4732
3240.4904
3240.4951
3240.4965
3240.7167
3240.7260
3240.8262
3240.9361
3253.5196
3253.5290
3253.7490
3253.7605

3.3892
49.0374
21.2733
$0.000 \odot$
12.8779
154.3462
35.7962
176.0399
11.2974
9.7603
79.0456
$0.000 \odot$
230.9001
$0.000 \odot$
0.6573
$0.000 \odot$
82.9819
50.8214
3.0397
$0.000 \odot$
37.5333
$382.135 \odot$
14.1503
6.3246
52.4036
$0.00 \odot \odot$
12.7737
71.5880
2.8961
$0.00 \odot \odot$
39.7340
135.0383
1.1548
$0.000 \odot$

